TITLE: Emittance Growth of an Nonequilibrium Intense Electron Beam in a Transport Channel with Discrete Focusing

AUTHOR(S): B. E. Carlsten

SUBMITTED TO:

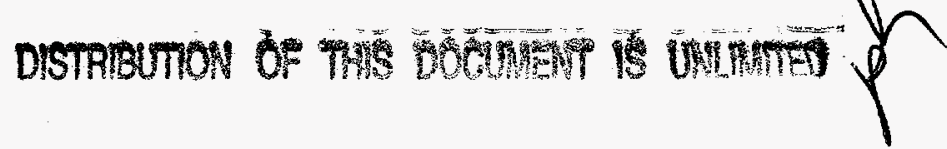

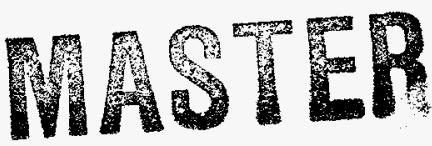

By acceptance of this article, the publisher recognizes that the U.S. Government retains a nonexclusive, royalty-free license to publish or reproduce the published form of this contribution, or to allow others to do so, for U.S. Government purposes.

The Los Alamos National Laboratory requests that the publisher identify this article as work performed under the auspices of the U.S. Department of Energy.

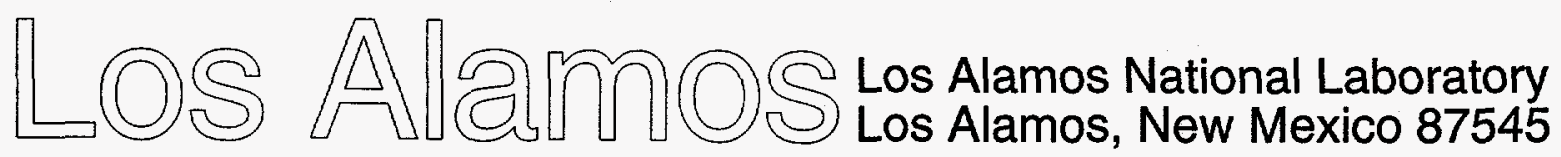




\section{DISCLAIMER}

This report was prepared as an account of work sponsored by an agency of the United States Government. Neither the United States Government nor any agency thereof, nor any of their employees, make any warranty, express or implied, or assumes any legal liabiity or responsibility for the accuracy, completeness, or usefulness of any information, apparatus, product, or process disclosed, or represents that its use would not infringe privately owned rights. Reference herein to any specific commercial product, process, or service by trade name, trademark, manufacturer, or otherwise does not necessarily constitute or imply its endorsement, recommendation, or favoring by the United States Government or any agency thereof. The views and opinions of authors expressed herein do not necessarily state or reflect those of the United States Government or any agency thereof. 


\section{DISCLAIMER}

Portions of this document may be illegible in electronic image products. Images are produced from the best available original document. 


\title{
Emittance Growth of an Nonequilibrium Intense Electron Beam in a Transport Channel with Discrete Focusing
}

\author{
Bruce Carlsten \\ Los Alamos National Laboratory \\ Los Alamos, NM 87545
}

\begin{abstract}
We analyze the emittance growth mechanisms for a continuous, intense electron beam in a focusing transport channel, over distances short enough that the beam does not reach equilibrium. The emittance grows from the effect of nonlinear forces arising from (1) current density nonuniformities, (2) energy variations leading to nonlinearities in the space-charge force even if the current density is uniform, (3) axial variations in the radial vector potential, (4) an axial velocity shear along the beam, and (5) an energy redistribution of the beam as the beam compresses or expands. The emittance growth is studied analytically and numerically for the cases of balanced flow, tight focusing, and slight beam scalloping, and is additionally studied numerically for an existing 6-MeV induction linear accelerator. Rules for minimizing the emittance along a beamline are established. Some emittance growth will always occur, both from current density nonuniformities that arise along the transport and from beam radius changes along the transport.
\end{abstract}


In this paper, we will analyze the emittance growth of a continuous, intense electron beam in a transport channel made up of short discrete solenoids or quadrupoles. We will assume that the transport channel is short with a few (up to maybe a dozen) discrete focusing elements, the beamline optics are designed so that the electron beam is focused to the smallest spot possible at the end of the channel, the focusing is not periodic, and the electron beam does not reach an equilibrium periodic phase-space distribution. In addition, we will assume that all elements are perfectly aligned and for most cases that the focusing elements are perfect with no fringe fields, which eliminates several very significant emittance growth mechanisms, such as the corkscrew mechanism [1-3] and radial aberrations in the focusing. (There will be one practical example in which the axial magnetic field will be expanded off axis, including the fringe fields). However, quite a few emittance growth mechanisms still exist based on nonlinear forces in both the space-charge force (even for a uniform density beam) and the externally applied focusing force, which are poorly understood and are becoming important for the new generation of high current, low emittance induction linear accelerators. We will consider the emittance growth for three cases: (1) balanced focusing (which sets a lower limit on the minimum possible emittance growth), (2) hard focusing, including the effects both from the final focus magnet itself and those occurring in the drift to the target from the magnet), and (3) gentle scalloping motion (which is the case for most of the transport channel where the beam is focused in the discrete focusing magnets and expands between them). The emittance will grow from nonlinear forces arising from (1) nonlinearities in the space-charge force, (2) axial variations in the radial vector potential, (3) energy fluctuations driven by the particles' radial motion, and (4) radial variations in the axial velocity. This is the first calculation of the effects from the axial variations in the radial vector potential; this effect is typically small compared to the other effects for a solenoidal focused transport, but it can become important for transport channels using quadrupole focusing. The numerical examples presented here will assume more-or-less nominal parameters for the Integrated Test Stand (ITS) [4], which is a 6-MeV, 4-kA, solenoidal focused beamline. The nominal normalized, $90 \%$ emittance is about $1000 \mathrm{~mm}$ 
$\mathrm{mrad}$, and the beam is typically about 2 to $3 \mathrm{~cm}$ in radius. Note that this means that the divergence of the beam is typically about $5 \mathrm{mrad}$, and that the effects from nonlinear forces can stay correlated for several meters.

The approach taken here is to assume that the beam density is initially uniform and to find nonlinearities in the beam divergence. This approach is fundamentally different than assuming that the beam distribution is in equilibrium [5]. The nonequilibrium assumption is justified because of the nonuniformity of the focusing of the beam down the beamline and the short overall transport distance. Our goal is to classify and quantify different emittance growth mechanisms. Analytic formulas will be used to estimate the emittance growth from specific causes, which will then be compared to direct numerical solutions. We will use these results to help interpret aspects of the emittance growth in the ITS beamline.

We will start by reviewing the emittance formalism used here. Next, we will construct the radial equation of motion including the effect of both the space-charge forces and the focusing forces. Finally, we will examine the emittance growth for the three cases outlined above, both analytically and numerically. We will find that the largest emittance growth contribution is generated in the final focus solenoid and following drift, which is because the beam size is large in the final focus magnet in order to get the smallest possible spot size at the target and because the resulting beam convergence angle is the largest. If the final focus magnet is a solenoid, the major emittance growth mechanism results from the variation in axial velocity radially along the beam, and can lead to a relatively large emittance growth. If the final focus magnet is a quadrupole, the dominant emittance growth mechanism is from the potential depression of the beam, which leads to a variation in the focal length of the quadrupole. However, the effect from the potential depression is partially canceled by the axial variation of the radial vector potential, and the resulting emittance growth is about a factor of three less, for the example given. There is also an emittance growth in the drift between the focusing element and the target from the change in the particles' energies from the radial convergence of the beam. In addition, very large emittance growths can result from mismatches within solenoidal fields and from radial density variations. 


\section{Emittance Formalism}

To be consistent with the experimental measurements, we will use the so-called $90 \%$ normalized emittance, which, for the radial coordinate, is given by

$$
\varepsilon=2 \gamma \sqrt{\left\langle r^{2}\right\rangle\left\langle r^{\prime 2}\right\rangle-\left\langle r r^{\prime}\right\rangle^{2}} .
$$

We are going to evaluate the emittance growth of an initially zero-emittance beam in the following sections, for various focusing configurations. In the later analytic cases, we will assume that the beam density profile is uniform (so the $\left\langle r^{2}\right\rangle$ factor is trivial, and just equal to $r_{b}^{2} / 2$ where $r_{b}$ is the beam radius), and that the lowest order nonlinear force present will cause a nonlinearity in the radial divergence (linearly proportional to the length the beam is subjected to that force). Then, after some distance traversing the beamline, the radial divergence will be expressed as $r^{\prime}=\alpha r+f(r)$, where $\alpha$ is some constant and $f(r)$ is some (nonlinear) function.

Note that

$$
\left\langle r^{\prime 2}\right\rangle=\alpha^{2}\left\langle r^{2}\right\rangle+2 \alpha\langle r f(r)\rangle+\left\langle f(r)^{2}\right\rangle
$$

and

$$
\left\langle r r^{\prime}\right\rangle^{2}=\alpha^{2}\left\langle r^{2}\right\rangle^{2}+2 \alpha\left\langle r^{2}\right\rangle\langle r f(r)\rangle+\langle r f(r)\rangle^{2},
$$

and the emittance is

$$
\varepsilon^{2}=4 \gamma\left(\left\langle r^{2}\right\rangle\langle f(r)\rangle^{2}-\langle r f(r)\rangle^{2}\right)
$$


Thus only the nonlinear part of the induced radial expansion contributes to the emittance growth and we can ignore the linear part while calculating beam ensemble averages (this will make our emittance growth calculations easier).

Also note that the $90 \%$ emittance using either the horizontal or vertical ensemble averages is calculated slightly differently:

$$
\varepsilon=4 \gamma \sqrt{\left\langle x^{2}\right\rangle\left\langle x^{\prime 2}\right\rangle-\left\langle x x^{\prime}\right\rangle^{2}}
$$

The extra factor of two arises because

$$
\begin{aligned}
& \left\langle r^{2}\right\rangle=2\left\langle x^{2}\right\rangle \\
& \left\langle r^{\prime 2}\right\rangle=2\left\langle x^{\prime 2}\right\rangle
\end{aligned}
$$

for a cylindrically symmetric beam.

We will assume that any emittance growths we calculate in later sections add in quadrature to the initial emittance growth - thus the emittance growth is not the difference between the initial and final emittances. However, with this definition, the emittance growth is independent of the initial emittance. In Section IV, we will numerically consider correlations between different emittance growth mechanisms.

II. Derivation of the Radial Equation of Motion, including Nonlinear Focusing Forces

In this section, we wish to derive an expression for the radial equation of motion that we can use to examine various emittance growth mechanisms. For simplicity, we will only derive the radial equation of motion for a beam in a solenoid; after we derive it, we will discuss the differences introduced if the focusing is from a quadrupole instead. In particular, we wish to have an expression we can use to estimate the emittance growth 
from both the space-charge and solenoidal fields, for the cases of (1) matched flow (no net radial force nor radial motion), (2) strong focusing (where the solenoidal force is much larger than the space-charge force - for example, this is the case for the final focus solenoid which focuses the beam to a very small spot at the target), and (3) scalloped flow, in which the electron beam is nearly matched, but is radially oscillating. Scalloped flow will occur in any beamline where the focusing is provided by several small solenoids, each separated by a relatively large distance.

The approach we will take will be to consider the radial equation of motion for a particle, and to expand the force terms for a specific particle around the force seen by a nominal particle at the beam's center. We will then assume that each particle experiences the same force over some axial distance $l$ as they drift, and that the resulting rms spread in the radial divergence will be given by $l$ times the rms spread in the radial force. Once this is done for the various cases described above, the emittance growth can be easily evaluated by performing the ensemble averages in Eqn. (4). Since we are interesting in the emittance growth from nonlinear components of the space-charge and focusing forces, we will expand the radial equation of motion carefully.

\section{A. Radial Force Equation}

The radial equation of motion for a particle within the beam within the central part of a solenoid (where the applied magnetic field from the solenoid is purely axial) is given by

$$
m \frac{d(y \dot{r})}{d t}=e E_{r}+e\left(v_{\theta} B_{\text {dia }}-v_{z} B_{\theta}\right)+e v_{\theta} B_{e x t}+y m v_{\theta}^{2},
$$

where $\gamma$ is the relativistic mass factor, $B_{\text {ext }}$ is the axial magnetic field from the solenoid (including the diamagnetic effect from the image currents in the beampipe), $B_{d i a}$ is the induced diamagnetic axial magnetic field from the beam current opposing the solenoidal field, $B_{\theta}$ is the azimuthal magnetic field from the space charge, and $E_{r}$ is the radial 
electric field from the space charge, all at the position of the particle, and $e$ and $m$ are the electronic charge and mass, respectively. Most of these terms are mostly linear with radius. For balanced flow (the beam edge is at a constant radius), the linear components cancel. The $v_{z} B_{\theta}$ term mostly cancels $E_{r}$ (to order $1 / \gamma^{2}$ ), but also includes a centrifugal space-charge effect that we approximate later. For balanced flow, the solenoid strength is adjusted such that the linear part of the combination of $e v_{\theta} B_{e x t}$ and the centrifugal force will cancel the linear part of the resulting space-charge force. There is also a potential depression within the beam (a variation of $\gamma$ that is a function of radius). Our approach will be to expand the radial equation of motion in terms of the variation of $\gamma$, to lowest order, which we will then use to estimate the emittance growth for various cases.

The azimuthal velocity is found by application of Busch's Theorem [6] (the conservation of angular momentum):

$$
v_{\theta}=\frac{e\left(B_{e x t}+B_{d i a}\right)}{2 \gamma m} r .
$$

We will assume that any radial divergence of the beam is small, and use Gauss' Law to find the radial electric field,

$$
E_{r}(r)=\int_{0}^{r} \frac{\rho(v) v}{\varepsilon r} d v
$$

where $\rho$ is the charge density and $v$ is a dummy variable for the radial integration. The diamagnetic field is given by

$$
B_{d i a}=\int_{r}^{r_{b}} \frac{\mu e B \rho(v) v}{2 \gamma m} d v
$$


where $B$ is the full axial magnetic field (applied plus diamagnetic) and where now $r_{b}$ is the radial edge of the beam. The relativistic mass factor is given by

$$
\gamma(r)=\gamma_{a}+\gamma_{1}(r)=\gamma_{a}+\int_{0}^{r} \frac{e E_{r}(v)}{m c^{2}} d v
$$

where $\gamma_{a}$ is the mass factor along the axis $(r=0)$. Let us assume that the space-charge density is of the form $\rho=\rho_{o} r^{n}$. Explicit evaluation of the above integrals for this charge density profile give

$$
\begin{aligned}
& E_{r}=\frac{\rho_{o}}{\varepsilon(n+2)} r^{n+1} \\
& \gamma_{1}=\frac{e}{m c^{2}} \frac{\rho_{o}}{\varepsilon(n+2)^{2}} r^{n+2}=\frac{e E_{r}}{m c^{2}} \frac{r^{n+2}}{n+2} \\
& \frac{B_{d i a}}{B}=\frac{\mu e}{2 m \gamma} \frac{\rho_{o}}{(n+2)}\left(r_{b}^{n+2}-r^{n+2}\right)=\left(\frac{\gamma_{b}}{\gamma_{a}}-\frac{\gamma_{1}}{\gamma_{a}}\right) \frac{n+2}{2}
\end{aligned}
$$

where we have now introduced $\gamma_{b}$ as the difference in the relativistic mass factor between the center and the radial edge of the beam, and substituted in $\gamma_{a}$ for $\gamma$ in the expression for the diamagnetic field (we will see that this is correct a little later). We can manipulate the axial field components in order to find a more useful form. The total magnetic field in terms of the diamagnetic component and the total externally applied field $B_{e x t}$ is given by

$$
B=B_{\text {ext }}-\frac{B_{\text {dia }}}{B} B
$$

or, to lowest order, 


$$
B=B_{\text {ext }}\left(1-\frac{B_{\text {dia }}}{B}\right)=B_{\text {ext }}\left(1-\frac{\gamma_{b}}{\gamma_{a}}\left(\frac{n+2}{2}\right)+\frac{\gamma_{1}}{\gamma_{a}}\left(\frac{n+2}{2}\right)\right)=B_{a}\left(1+\frac{\gamma_{1}}{\gamma_{a}}\left(\frac{n+2}{2}\right)\right)
$$

where now the total axial magnetic field along the axis $(r=0)$ is given by

$$
B_{a}=B_{e x t}\left(1+\frac{\gamma_{b}}{\gamma_{a}}\left(\frac{n+2}{2}\right)\right)^{-1}
$$

These are particularly useful forms to use, because if the charge density is uniform $(n=0)$,

$$
\begin{aligned}
& B=B_{a}\left(1+\frac{\gamma_{1}}{\gamma_{a}}\right) \\
& \gamma=\gamma_{a}\left(1+\frac{\gamma_{1}}{\gamma_{a}}\right)
\end{aligned}
$$

and $\gamma_{1}$ depends quadratically on the beam radius, and is positive (see Eqn. (12)). Also, if the charge density is uniform, Eqns. (16) are exact - this is easy to see by just substituting them into Eqn. (10), and using $B_{a}=B_{e x t}\left(1+\frac{\gamma_{b}}{\gamma_{a}}\right)^{-1}$.

Note that the azimuthal velocity in terms of the magnetic field on axis and the relativistic mass factor on axis is given by

$$
v_{\theta}=\frac{e B_{a} r}{2 \gamma_{a} m}\left(\frac{1+\frac{\gamma_{1}}{\gamma_{a}} \frac{n+2}{2}}{1+\frac{\gamma_{1}}{\gamma_{a}}}\right) \text {. }
$$

If the space-charge density is uniform $(n=0)$, the term in the parenthesis is unity, and the azimuthal velocity is radially linear. 
The beam-induced azimuthal magnetic field in Eqn. (7) is given in terms of the vector potential by

$$
B_{\theta}=\frac{\partial}{\partial z} A_{r}+\frac{1}{r} \frac{\partial}{\partial r} r A_{z}
$$

Note that

$$
\begin{aligned}
& \phi=\int \frac{\rho}{4 \pi \varepsilon r} d \vec{r} \\
& \vec{A}=\int \mu \frac{\vec{j}}{4 \pi r} d \vec{r}
\end{aligned}
$$

where $\phi$ is the scalar potential, $\vec{j}$ is the current density, and the integrals are over all space. The current density is

$$
\vec{j}=\rho \beta c(\hat{z} \cos \Psi+\hat{r} \sin \Psi)
$$

where the angle $\Psi$ is given by

$$
\Psi=\frac{r}{r_{b}} \frac{d r}{d z}
$$

Note that the difference between the azimuthal vector potential and $(\beta / c) \phi$ is on the order of $\Psi^{2}$, but the radial vector potential scales as $(\beta / c) \phi$ times $\Psi$. Because $\Psi^{2}$ is much smaller than $1 / \gamma^{2}$, this means that the space-charge electric field and the spacecharge magnetic field from the axial vector potential still cancel to order $1 / \gamma^{2}$ to first order in $\Psi$, and the additional space-charge force introduced by the radial vector potential adds in a force of order $\Psi$ that is not canceled by other fields. 
The vector potential for a uniform density beam at a location $y$ along the vertical axis is given by

$$
\vec{A}=\int_{-z}^{z} d \zeta \int_{0}^{2 \pi} d \theta \int_{0}^{r_{b}} \mu \frac{I(\sin \theta \sin \Psi, 0, \cos \Psi)}{4 \pi^{2} r_{b}^{2} \sqrt{\zeta^{2}+r^{2}+y^{2}-2 r y \sin \theta}} r d \vec{r}
$$

where additionally the beam radius is given as a function of axial position from the observer location $\zeta$ by

$$
r_{b}=r_{o}+\zeta \tan \Psi
$$

and where $r_{o}$ is the beam radius at the axial position of the observer location.

As is well known [7], the integral for the axial vector potential diverges as the limit of integration $z$ approaches infinity - this, however, is not the case for the radial vector potential, which quickly reaches a maximum. This is easily understood by observing that for large axial displacements, the radical part of the denominator in Eqn. (22) becomes $\sqrt{\zeta^{2}}$ and the $\sin \theta$ term in the numerator then integrates to zero (far from the observer location, the different radial velocities of the beam current density average to zero).

We can normalize the radial vector potential to the scalar potential at the beam radius $\phi_{b}=\gamma_{b} m c^{2} / e$,

$$
A_{r}=\frac{d r}{d z} \chi \frac{\beta}{c} \phi_{b}=\frac{d r}{d z} \chi \mu \frac{I}{4 \pi}
$$

where now $\chi$ is a parameter between zero and unity, depending on the beam geometry and divergence.

In Fig. 1 we plot $\chi(d r / d z)$ at the beam radius versus the axial limit of integration, normalized to the beam radius, for a divergence of $10 \mathrm{mrad}$. We see that the 
radial vector potential does indeed quickly reach its asymptotic value (for an axial limit of integration of about 10 beam radii), and that $\chi \approx 0.5$. In Fig. 2 , we plot the asymptotic value of $\chi(d r / d z)$ as a function of radial position within the beam. The dependence of radial position is less than linear, but we will assume a linear dependence for emittance calculations later. In Fig. 3, we plot the asymptotic value of $\chi(d r / d z)$ as a function of beam edge divergence, and see that $\chi \approx 0.5$ for a wide range of beam edge divergences.

For small rates of change of the beam edge divergence, we find

$$
B_{\theta}=-\frac{\beta}{c} \frac{1}{r} \frac{\partial}{\partial r}(r \phi)-\frac{\beta}{c} \frac{r}{r_{b}} \frac{d^{2} r}{d z^{2}} \phi_{b} \chi
$$

At this point, we have written out all the terms on the right-hand-side of the radial equation of motion, and we can evaluate the nonlinear terms. For emittance analysis, we want the radial divergence instead of the radial velocity, so we still need to change the variable of differentiation on the left-hand-side of the radial equation of motion. Using dots to refer to time derivatives and primes to refer to axial derivatives, we have

$$
\frac{d}{d t} \dot{r}=\dot{r} \frac{d \gamma}{d t}+\ddot{\gamma} \ddot{r}=\frac{e E_{r}}{m c^{2}} \dot{r}^{2}+\ddot{\gamma r}
$$

and

$$
\begin{aligned}
& \dot{r}=r^{\prime} v_{o}\left(1+\frac{v(r)}{v_{a}}\right) \\
& \ddot{r}=r^{\prime \prime} v_{z}^{2}(r)=r^{\prime \prime} v_{a}^{2}\left(1+2 \frac{v(r)}{v_{a}}\right)
\end{aligned}
$$

where we are defining an average axial velocity $v_{a}$ and a relative axial velocity $v$ to be

$$
v_{z}(r)=v_{a}+v(r)
$$


In a later section, we will show that for uniform focused flow, $v$ actually vanishes (as in the case for Brillouin flow for tenuous electron beams). After combining the focusing term and the centrifugal acceleration term, and combining the $r^{\prime \prime}$ terms and dividing through by a factor of $\gamma$, Eqn. (7) becomes

$$
r^{\prime \prime} m v_{o}^{2}\left(1+2 \frac{v}{v_{a}}-\frac{\gamma_{b}}{\gamma_{a}} \chi \frac{r}{r_{b}}\right)=\frac{e E_{r}}{\gamma \gamma^{*}(r)^{2}}-\frac{e^{2} B^{2} r}{4 \gamma^{2} m}-\frac{e}{c^{2} \gamma} v_{o}^{2} E_{r} r^{\prime 2}
$$

To lowest order in the small quantities, the radial force equation becomes in terms of the parameters evaluated on axis

$$
\begin{aligned}
r^{\prime \prime}= & {\left[\frac{e E_{r}}{m v_{a}^{2} \gamma_{a} \gamma^{*}(r)^{2}}\left(1-\frac{\gamma_{1}}{\gamma_{a}}\right)-\frac{e^{2} B_{a}^{2} r}{4 \gamma_{a}^{2} m^{2} v_{a}^{2}}\left(1+2 \frac{\gamma_{1}}{\gamma_{a}}\left(\frac{n+2}{2}-1\right)\right)-\frac{e}{\gamma_{a} m c^{2}} E_{r} r^{\prime 2}\right] } \\
& \times\left(1-2 \frac{v}{v_{a}}+\frac{\gamma_{b}}{\gamma_{a}} \chi \frac{r}{r_{b}}\right) .
\end{aligned}
$$

After a drift of length $l$, we find that the radial divergence is given by

$$
\begin{aligned}
r^{\prime}= & {\left[\frac{e E_{r}}{m v_{a}^{2} \gamma_{a} \gamma^{*}(r)^{2}}\left(1-\frac{\gamma_{1}}{\gamma_{a}}\right)-\frac{e^{2} B_{a}^{2} r}{4 \gamma_{a}^{2} m^{2} v_{a}^{2}}\left(1+2 \frac{\gamma_{1}}{\gamma_{a}}\left(\frac{n+2}{2}-1\right)\right)-\frac{e}{\gamma_{a} m c^{2}} E_{r} r_{o}^{\prime 2}\right] } \\
& \times\left(1-2 \frac{v}{v_{a}}+\frac{\gamma_{b}}{\gamma_{a}} \chi \frac{r}{r_{b}}\right)+r_{o}^{\prime}
\end{aligned}
$$

where $r_{o}^{\prime}$ is the initial radial divergence, which we will assume to be radially linear, and is therefore uninteresting and we will not carry this term further. We also have to be careful with our interpretation of $r^{\prime}$ in this equation - the $r_{o}^{\prime}$ that is on the right-hand-side of the equation is the average rate of divergence (or convergence) of the beam (which we will assume to be linear to first order). The $r^{\prime}$ on the left-hand-side of the equation is the change of the divergence. 
Now note that the focal length of a solenoid for the beam near the axis is defined by

$$
f=\frac{4 \gamma_{a}^{2} m^{2} v_{a}^{2}}{l e^{2} B_{a}^{2}}
$$

and so the radial divergence after a length $l$ becomes

$$
\begin{aligned}
r^{\prime}= & {\left[\frac{l e E_{r}}{m v_{a}^{2} \gamma_{a} \gamma^{*}(r)^{2}}\left(1-\frac{\gamma_{1}}{\gamma_{a}}\right)-\frac{r}{f}\left(1+2 \frac{\gamma_{1}}{\gamma_{a}}\left(\frac{n+2}{2}-1\right)\right)-\frac{l e}{\gamma_{a} m c^{2}} E_{r} r_{o}^{\prime 2}\right] } \\
& \times\left(1-2 \frac{v}{v_{a}}+\frac{\gamma_{b}}{\gamma_{a}} \chi \frac{r}{r_{b}}\right)
\end{aligned}
$$

B. Special Case of Uniform Charge Density

If the charge density is uniform, the radial divergence becomes

$$
r^{\prime}=\left[\frac{l e E_{r}}{m v_{a}^{2} \gamma_{a} \gamma^{*}(r)^{2}}\left(1-\frac{\gamma_{1}}{\gamma_{a}}\right)-\frac{r}{f}-\frac{l e}{\gamma_{a} m c^{2}} E_{r} r_{o}^{\prime 2}\right]\left(1-2 \frac{v}{v_{a}}+\frac{\gamma_{b}}{\gamma_{a}} \chi \frac{r}{r_{b}}\right) .
$$

Note that the effect of the potential depression of the beam exactly cancels the effect of the diamagnetic effect, leading to a purely linear focusing force. In the following sections, we will mostly assume that the charge density is uniform. For a quadrupole, this equation is changed simply by substituting $(r / f)\left(1-\gamma_{1} / \gamma_{a}\right)$ for $r / f$.

\section{Emittance Growth Estimates}

In this section, we will consider the emittance growths for the balanced flow case, the hard focusing case (from the final focus magnet to the target spot size), and the gentle 
scalloping case. For all these cases, we will consider a uniform density electron beam the emittance growth from density nonlinearities will be discussed in the balanced uniform flow case, and is in general less interesting. These emittance growths arise from nonlinearities in the introduced radial divergence (Eqn. (34)) - in particular, from (1) the $\gamma_{1} / \gamma_{a}$ term multiplying the space-charge force, (2) the $r_{o}^{\prime 2}$ term, (3) the $v / v_{o}$ term, and (4) the $\chi \gamma_{b} / \gamma_{a}$ term (for quadrupole focusing this term becomes to lowest order $\left.\gamma_{1} / \gamma_{a}-\chi \gamma_{b} / \gamma_{a}\right)$. The emittance growth from (1) is physically due to a nonlinearity in the radial space charge force. The emittance growth from (2) arises from the fact that even if the radial momentum change is linear, if particles at different radii gain or lose energy at different rates, this will lead to a nonlinearity in the radial equation of motion. Issue (3) leads to a different amount of time particles at different radii spend inside the solenoid. The physical explanation for (4) is more complicated and has to do with the fact that the energy in the Coulomb fields associated with the particles has inertia.

We will assume that the focusing elements are very thin for simplicity. In the emittance growth formulas that we derive later, the focusing element length is important only for the case of the variation of axial velocities in a solenoid. As a result, we will implicitly assume that the quadrupoles are very thin and that quadrupole doublets are used for two-plane focusing. In this case, the correct emittance growths are found by using the net beam convergence and focusing focal length in the following formulas. The only errors that arise are (1) from the neglect of potentially large beam divergences between the quadrupoles in the doublet and (2) from the neglect of fringe field aberrations in the quadrupoles' focusing fields.

These emittance growth estimates are unable to address the issue of correlations between different mechanism, which will be examined in the following section. If the effects are completely uncorrelated, we expect that the emittance growths add in quadrature. If the effects are correlated, the emittance growths can either add or subtract, depending on the nature of the correlation.

\section{A. Balanced Uniform Flow (Uniform Charge Density)}


In this section, we will assume that the focusing just balances the radial space charge force (for simplicity we will assume near the axis of the beam), and that $r_{o}^{\prime}=0$.

In this case, the third term within the first parenthesis vanishes, and any nonlinearity introduced in the radial divergence comes from the nonlinearity in the first term within the first parenthesis (all nonlinear effects from terms within the second parenthesis are second order).

Balanced flow means that

$$
\frac{l e E_{r}}{v_{a}^{2} \gamma_{a}^{3}}=\frac{r}{f}=\frac{l e^{2} B_{a}^{2} r}{4 \gamma_{a}^{2} m v_{a}^{2}}
$$

There is clearly a nonlinearity in the space-charge force associated with the beam's potential depression, but it is instructive to also investigate the linearity of the $\gamma^{*}$ factor in the space-charge term.

The space-charge radial electric field at a radial position $r$ from a ring at radius $v$ is given by

$$
d E_{r}=\frac{\rho_{o} v}{\varepsilon r} d v
$$

The radial force is at $r$ is then given by

$$
F_{r}(r)=\int_{0}^{r} \frac{e \rho_{o} v}{\varepsilon r}(1-\beta(r) \beta(v)) d v
$$

which is easily evaluated as soon as we have an expression for the radial velocity as a function of radial position.

The axial velocity is found from the conservation of energy: 


$$
1-\beta_{z}^{2}-\beta_{\theta}^{2}=\frac{1}{\gamma^{2}}=\frac{1}{\gamma_{a}^{2}}\left(1-\frac{2 \gamma_{1}(r)}{\gamma_{a}}+\frac{3 \gamma_{1}(r)^{2}}{\gamma_{a}^{2}}\right),
$$

where, as before, $\gamma_{a}$ is the normalized beam energy along the axis and $\gamma_{1}(r)$ is the beam energy change from the axis, and we have kept this equation to second order in $\gamma_{1} / \gamma_{a}$. From before, we know that the azimuthal velocity is given by

$$
\beta_{\theta}^{2}=\left(\frac{e B(r) r}{2 \gamma m c}\right)^{2}=\left(\frac{e B_{a} r}{2 \gamma_{a} m c}\right)^{2}=2 \frac{\gamma_{1}}{\gamma_{a}^{3}},
$$

using the condition of balanced flow (Eqn. (35)) and the relation between the radial electric field and the potential depression for a uniform beam. Using this expression in Eqn. (38), we find

$$
1-\beta_{z}^{2}=\frac{1}{\gamma_{a}^{2}}\left(1+\frac{3 \gamma_{1}^{2}}{\gamma_{a}^{2}}\right),
$$

and any variation in the axial beam velocity is second order in the small quantities. Thus the entire beam essentially has the same axial velocity, and indeed $\gamma^{*}=\gamma_{a}$, to first order in $\gamma_{1} / \gamma_{a}$. This is a more general case of the same well-known effect for Brillouin flow for tenuous electron beams [6].

In order to estimate the emittance growth from the nonlinear contribution from the radial space-charge force, we start with the nonlinear part of Eqn. (34),

$$
r^{\prime}=\frac{l e E_{r}}{m v_{a}^{2}} \frac{\gamma_{1}}{\gamma_{a}^{4}}
$$


In terms of the beam current, the radial electric field is given by

$$
E_{r}=\frac{I r}{2 \pi \varepsilon_{b}^{2} v_{a}}
$$

Using the definition of the Alfven current $\left(I_{A}=4 \pi \varepsilon m c^{3} / e\right)$, we can rewrite the beam divergence as

$$
r^{\prime}=2 \frac{I^{2}}{I_{A}^{2}} \frac{1}{\beta^{4} \gamma_{a}^{4}} \frac{l r^{3}}{r_{b}^{4}}
$$

Note that the second moments of a divergence of the form $r^{\prime}=\kappa r^{3}$ are:

$$
\begin{aligned}
& \left\langle r^{2}\right\rangle=\frac{r_{b}^{2}}{2} \\
& \left\langle r^{\prime 2}\right\rangle=\kappa^{2} \frac{r_{b}^{6}}{4} \\
& \left\langle r r^{\prime}\right\rangle=\kappa \frac{r_{b}^{4}}{3}
\end{aligned}
$$

and the normalized, $90 \%$ emittance is

$$
\varepsilon=\frac{2 \gamma_{a} \beta}{\sqrt{72}} \kappa r_{b}^{4}
$$

For this case, then,

$$
\kappa=2 \frac{l}{r_{b}^{4}} \frac{1}{\beta^{4} \gamma_{a}^{4}} \frac{I^{2}}{I_{A}^{2}}
$$

and the emittance induced from the space-charge force nonlinearities is 


$$
\varepsilon=l \frac{4}{\sqrt{72}} \frac{1}{\beta^{3} \gamma_{a}^{3}} \frac{I^{2}}{I_{A}^{2}}
$$

For a 4-kA beam at $6 \mathrm{MeV}$, the emittance growth is about $l \times 20 \mathrm{~mm} \mathrm{mrad}$.

Note that for a quadrupole, there are two differences - first, both the space-charge force and the focusing force have a $1-\gamma_{1} / \gamma_{a}$ dependency, and thus they cancel exactly to lowest order, and second, the axial velocity is now not uniform. However, the emittance growth from the variation in the axial velocity is higher order (the maximum variation in the axial velocity from the potential depression is $c \gamma_{1} / \beta \gamma_{a}^{3}$ ), and the resulting emittance growth is lower than that for solenoidal focusing, Eqn. (47).

The emittance growth from a nonuniformity in the space-charge density of the order of $\gamma_{b} / \gamma_{a}$ will be of a similar size as that shown in Eqn. (47). Equation (47) can be scaled to estimate the emittance growth from either a larger or smaller density variation.

\section{B. Hard Focusing Case (Uniform Charge Density)}

Next, let us consider the case where the beam is focused very strongly by a magnet, such as in the case of the final focus. There are two regimes in this case - first, inside the magnet itself, and second in the drift after the magnet.

The minimum rms spot size a distance $L$ after a lens is given by

$$
r_{\min }=\sqrt{2 L \varepsilon_{o, u n}\left(\frac{\Delta \gamma}{\gamma}\right)_{m s}}
$$

where $\varepsilon_{o, u n}$ is the unnormalized emittance at the target and $(\Delta \gamma / \gamma)_{r m s}$ is the rms energy spread at the lens [8]. The minimum spot size arises when the initial rms spot size at the lens is given by 


$$
r_{\text {lens }}=\sqrt{\frac{L \varepsilon_{o, u n}}{\left(\frac{\Delta \gamma}{\gamma}\right)_{m m s}}},
$$

which is in general fairly large, leading to relatively large emittance growths. Note that the emittance at the lens $\varepsilon_{0}$ has to include both the emittance before the lens, the emittance growth in the lens, and the emittance growth in the drift between the lens and the target.

\section{Effects inside the solenoid}

As before, we will first evaluate the emittance growth for a solenoid, and then discuss the differences for a quadrupole. There are two effects worth considering inside the solenoid. First, it is instructive to estimate the emittance growth from the axial change in the radial vector potential within the final focus solenoid itself. Second, we need to look at the effect of the spread of the axial velocity, which occurs if the final focus magnet is a solenoid. The nonlinear radial divergence induced in the solenoid from the axial change of the radial vector potential is of the form

$$
r^{\prime}=\lambda r^{2},
$$

where now $\lambda=\chi \gamma_{b} / f r_{b} \gamma_{a}$. Note that

$$
\begin{aligned}
& \left\langle r^{2}\right\rangle=\frac{r_{b}^{2}}{2} \\
& \left\langle r^{\prime 2}\right\rangle=\frac{r_{b}^{4}}{3} \lambda^{2} \\
& \left\langle r^{\prime}\right\rangle=\frac{2 r_{b}^{3}}{5} \lambda
\end{aligned}
$$

and the normalized emittance is 


$$
\varepsilon=2 \gamma_{a} \beta \frac{r_{b}^{2} \lambda}{\sqrt{150}}=\frac{2}{\sqrt{150}} \frac{r_{b}^{2}}{f} \chi \frac{I}{I_{A}}
$$

where we have used $\gamma_{b}=I / \beta I_{A}$. For the case of the ITS final focus solenoid, $\gamma_{a}=12.74, l=0.1 \mathrm{~m}, f=0.6 \mathrm{~m}, r_{b}=0.03 \mathrm{~m}$, and the emittance growth is about $30 \mathrm{~mm} \operatorname{mrad}$ (using $\chi \approx 0.5$ ).

Now let us consider the effect from the spread in the axial velocity of the beam within the solenoid. The variation in the axial velocity $\left(v_{z}=v_{a}+v\right)$ is given by

$$
v=-\frac{\gamma_{b}}{\gamma_{a}^{3}} \frac{c^{2}}{v_{a}}-\frac{c^{2}}{2 v_{a}} \beta_{r}^{2}-\frac{c^{2}}{2 v_{a}} \beta_{\theta}^{2}
$$

where $v_{a}$ is the axial velocity at the center of the beam. Inside the solenoid, the azimuthal velocity term dominates, and we find

$$
v=-\frac{c^{2}}{2 v_{a}} \beta_{\theta}^{2}=-\frac{e^{2} B^{2} r^{2}}{4 \gamma^{2} m^{2} v_{a}}
$$

This leads to the divergence in the beam introduced by the solenoid (ignoring all terms except for the terms depending on the solenoid's focal length) as

$$
r^{\prime}=-\frac{r}{f}\left(1-2 \frac{v}{v_{a}}\right)=-\frac{r}{f}\left(1-2 \frac{r^{2}}{l f}\right)
$$

The normalized, $90 \%$ emittance growth from the nonlinear part of this divergence is now given by Eqn. (45) $\left(\kappa=2 / l f^{2}\right)$,

$$
\varepsilon=\frac{\sqrt{2}}{3} \gamma_{a} \frac{r_{b}^{4}}{l f^{2}}
$$


For the case of the ITS final focus solenoid, the emittance growth is about $135 \mathrm{~mm} \mathrm{mrad}$.

For a quadrupole, the emittance growth from the variation in the axial velocity is negligible as long as the quadrupole is short compared to the drift afterwards (it is the same as will be found in the next section for the drift after the solenoid). However, the nonlinearity in the divergence is now given by $r / f\left(1-\gamma_{1} / \gamma_{a}+\chi r \gamma / r_{b} \gamma_{b}\right)$. Note that the effect of the axial variation of the radial vector potential tends to partially cancel the nonlinearity from the effect of the beam's potential depression on the focusing. Physically, this is similar to the net cancellation of the centrifugal space-charge force and the potential depression in a dipole field $[9,10,11]$. The emittance growth contribution from the vector potential is the same as was shown in Eqn. (52). The emittance growth from the potential depression itself can be easily evaluated using Eqn. (45) and $\kappa=I / \beta \gamma_{a} I_{A} f r_{b}^{2}$, which leads to a normalized, $90 \%$ emittance growth of

$$
\varepsilon_{\text {quad }}=\frac{2}{\sqrt{72}} \frac{I}{I_{A}} \frac{r_{b}^{2}}{f},
$$

or about $85 \pi \mathrm{mm} \mathrm{mrad}$ for the parameters we are considering. However, due to the opposition of these forces, this emittance growth and that from the radial vector potential (Eqn. (52)) do not add in quadrature, and there is in fact a fair amount of cancellation, so the overall emittance growth is about $1 / 3-1 / 2$ of that predicted by Eqn. (57) itself, or about $30-50 \mathrm{~mm}$ mrad (about $1 / 3$ that from using a solenoid). This is easy to show by directly comparing the emittance growth for a nonlinear divergence of $r^{\prime}=r^{3}-r^{2} r_{b} / 2$ to that for a nonlinear divergence of $r^{\prime}=r^{3}$. However, also note that the emittance growth from the variation in axial velocities within the solenoid scale as the beam radius to the fourth power, and it can be made smaller than the emittance growth in a quadrupole by decreasing the beam size in the solenoid sufficiently.

Effects in the drift after the solenoid 
For this case, the divergence term in Eqn. (34) dominates, but we need to be a little careful because the beam radius is changing over the axial range we are interested in. The growth of the radial divergence is given by

$$
d r^{\prime}=\frac{e E_{r}}{\gamma_{a} m c^{2}} \frac{r^{2}}{L^{2}} d l
$$

where $L$ is the distance from the solenoid to the final focus. We can rewrite the divergence in terms of simpler parameters as

$$
d r^{\prime}=2 \frac{I}{\gamma_{a} \beta I_{A}} \frac{r^{3}}{r_{b}^{2} L^{2}} d l
$$

and the emittance is given by Eqn. (45) $\left(\kappa=\left(2 I / \gamma_{a} \beta I_{A} r_{b}^{2} L^{2}\right) d l\right)$,

$$
d \varepsilon=d l \frac{4}{\sqrt{72}} \frac{r_{b}^{2}}{L^{2}} \frac{I}{I_{A}}
$$

This expression is easy to integrate, using $r_{b}=l r_{\text {lens }} / L$, using $r_{\text {lens }}$ as the beam radius at the solenoid, and we find the emittance at the final focus to be

$$
\varepsilon=\frac{4}{3 \sqrt{72}} \frac{r_{\text {lens }}^{2}}{L} \frac{I}{I_{A}}
$$

For the previous parameters and with an initial beam radius $r_{l e n s}$ of $3 \mathrm{~cm}$ and a final focus length $L$ of $60 \mathrm{~cm}$, the emittance growth is about $60 \mathrm{~mm} \mathrm{mrad}$.

It is easy to show that the effect from the spread in the axial velocities is unimportant in the drift. For the hard focusing case, the middle term on the right-handside dominates in Eqn. (53), and 


$$
v=-\frac{c}{2 \beta}\left(\frac{d r_{b}}{d z}\right)^{2} \frac{r^{2}}{r_{b}^{2}}
$$

As the beam drifts to the focus after the lens, the radial divergence is given by

$$
r^{\prime}=\left[\frac{l e E_{r}}{m v_{a}^{2} \gamma_{a} \gamma^{*}(r)^{2}}\left(1-\frac{\gamma_{1}}{\gamma_{a}}\right)-\frac{l e}{\gamma_{a} m c^{2}} E_{r} r_{o}^{\prime 2}\right]\left(1-2 \frac{v}{v_{a}}+\frac{\gamma_{b}}{\gamma_{a}} \chi \frac{r}{r_{b}}\right),
$$

which is after keeping terms to lowest order

$$
r^{\prime}=\frac{l e E_{r}}{m v_{a}^{2} \gamma_{a} \gamma^{*}(r)^{2}}\left(1-\frac{\gamma_{1}}{\gamma_{a}}+\frac{c}{v_{a} \beta} r_{o}^{\prime 2} \frac{r^{2}}{r_{b}^{2}}+\frac{\gamma_{b}}{\gamma_{a}} \chi \frac{r}{r_{b}}\right)-\frac{l e}{\gamma_{a} m c^{2}} E_{r} r_{o}^{\prime 2}
$$

We see that the nonlinear effect from the spread in axial velocities (the third term in the parenthesis) is on the order of $1 / \gamma^{* 2}$ smaller than the effect from the change in the relativistic mass factor (the last term), and thus can always be ignored. Also note that there is partial cancellation between the nonlinear radial dependence on $\gamma$ of the spacecharge term and the term arising from the change in the radial component of the vector potential (the second and fourth terms in the parenthesis).

\section{Gentle Scalloping Case (Uniform Charge Density)}

In general, the beam is not in completely balanced, uniform flow. The solenoids are discrete, and the beam gently undulates down the beamline. We can estimate the emittance growth for a length $l$ of scalloping motion, by using the divergence term in Eqn. (34) while assuming that the beam radius is a constant.

In this case, the accumulated nonlinear divergence after a length $l$ is given by 


$$
r^{\prime}=l \frac{e E_{r}}{\gamma_{a} m c^{2}} \frac{r^{2}}{r_{b}^{2}} \bar{\alpha}^{2}=2 \frac{I}{\gamma_{a} \beta I_{A}} \frac{l r^{3}}{r_{b}^{4}} \bar{\alpha}^{2}
$$

where now $\bar{\alpha}$ is the rms divergence of the scalloping of the radial beam edge (on the order of $10 \mathrm{mrad})$. For this case, $\kappa=2 I l \bar{\alpha}^{2} / \gamma_{a} \beta I_{A} r_{b}^{4}$, and the normalized, 90\% emittance growth is given by Eqn. (45),

$$
\varepsilon=\frac{4}{\sqrt{72}} l \frac{I}{I_{A}} \bar{\alpha}^{2}
$$

Note that the beam radius does not enter this equation. For the numbers used in the previous examples, the normalized, $90 \%$ emittance growth is about $l \times 15 \mathrm{~mm} \mathrm{mrad}$, which is comparable to the emittance growth from the nonlinear component of the spacecharge force at $6 \mathrm{MeV}$.

IV. Numerical Calculations of the Emittance Growth in a Channel with Solenoids

In this section, we use the simulation code SLICE to estimate the emittance growth for various focusing configurations, for comparison to the results found in the previous section and to determine the correlated effects between the various emittance growth mechanisms. We will also use this numerical tool to examine the emittance growth in the 6-MeV ITS machine. SLICE numerically integrates Eqn. (29) to evaluate the radial motion of a collection of particles; the space-charge field and the diamagnetic field is calculated at each axial step using the actual particle distribution. The logic in SLICE is arranged such that the contributions from the variation in axial velocities, from the radial divergence of the beam, and the axial divergence of the radial vector potential can all be calculated independently. 


\section{A. Balanced Uniform Flow (Uniform Charge Density)}

In Fig. 4a, we show the radial profile of the beam in a balanced flow configuration (the beam edge is defined in this and the following numerical plots as twice the rms beam size), for a $3-\mathrm{cm}$ radius, $6-\mathrm{MeV}, 4-\mathrm{kA}$ beam, with initially very low emittance. The applied external magnetic field for this case is 217 Gauss. In Fig. 4b, the emittance evolution as a function of axial location is shown. The emittance grows about $18 \mathrm{~mm}$ mrad per meter of drift, in excellent agreement with that predicted in the previous section, Section III.A. The emittance oscillates with a period of about $4.3 \mathrm{~m}$, showing that the correlation length is on the order of $2 \mathrm{~m}$, and that thermalization of the beam's transverse phase space takes significantly more than $8 \mathrm{~m}$ for these beam conditions. The oscillations in the emittance occur because the emittance growth is dominated by modifications in the radial particle distribution as the beam drifts, which are correlated with the individual particles' radial divergence, and are recoverable. This effect is essentially identical to emittance compensation schemes in rf photoinjectors [11].

\section{B. Hard Focusing Case (Uniform Charge Density)}

In this section, we examine the numerically calculated emittance growth for a beam focused to a very small focus, using the parameters discussed in Section III.B (6$\mathrm{MeV}$, 4-kA, 3-mm radius electron beam in a 10-cm long magnet with a focal length of 60 $\mathrm{cm})$.

For this case, we predicted an emittance growth of about $30 \mathrm{~mm}$ mrad from the axial variation of the radial vector potential in the solenoid, of about $135 \mathrm{~mm} \mathrm{mrad}$ from the variation in axial velocities in the solenoid, and of about $60 \mathrm{~mm} \mathrm{mrad}$ from the radial convergence of the beam in the drift between the solenoid and the focus.

Various SLICE outputs are shown for this case in Fig. 5. The beam radius is plotted in Fig. 5a. In this simulation, a solenoid with an axial field of $1.9 \mathrm{kG}$, and extending from the origin to $z=10 \mathrm{~cm}$, is used to focus the beam to a waist at about an axial position of $65 \mathrm{~cm}$. The magnetic field profile used (at the center of the beam) is 
shown in Fig. 5b. The average beam kinetic energy is shown in Fig. 5c, showing a slight energy depression as the beam is pinched. In Fig. 5d, we see the emittance evolution along the beam line (the initial emittance was $1 \mathrm{~mm}$ mrad), and in Fig. 5e we see the final (at an axial position of $1 \mathrm{~m}$ ) beam transverse configuration and phase space distribution. A curvature (and splitting) of the transverse phase space is seen.

From Fig. $5 \mathrm{~d}$ we see that the emittance growth within the solenoid is about $70 \mathrm{~mm}$ mrad, about half of that predicted by the analytic expressions. This happens because the effect from the axial variation in the radial vector potential counters the effect from the variation in the axial velocities, and because the emittance growth from the axial velocity variation is reduced as the beam becomes smaller within the solenoid itself. As the beam is focused to the waist, the nonlinear forces from the radial velocity (the $e v_{o}^{2} E_{r} r^{\prime 2} / c^{2} \gamma$ term in Eqn. (29) increase the emittance to about $120 \mathrm{~mm}$ mrad. The sharp dip right at the focus is from the compensation of the correlated radial velocity with radial position, as seen in the previous section. We can confirm that the radial velocity accounts for the subsequent emittance growth by modifying SLICE so that the radial velocity term is ignored in the evolution of the radial profile. The emittance growth for this case is shown in Fig. 5f, and indeed, there only a gradual emittance decrease through the focus until the location of the waist. The rapid increase in the emittance profiles in both Figs. $5 \mathrm{~d}$ and $5 \mathrm{f}$ is mostly due to the combination of the unwinding of the correlation that led to the decrease in the emittance at the waist and the redistribution of the particles' energies from the radial divergence, and only slightly on the bifurcation in the transverse shown in Fig. $5 e$.

\section{Gentle Scalloping Case (Uniform Charge Density)}

For this behavior, we will look at two cases - one where the scalloping is between a periodic array of thin solenoids, and one where the scalloping is due to a mismatch in an infinitely long solenoid.

In Fig. 6, we show the SLICE outputs for the periodic focusing case. In Fig. 6a, we see the radial profile, in Fig. $6 \mathrm{~b}$ we see the externally applied axial magnetic field, and 
in Fig. 6c we see the emittance evolution. In the last section, we predicted that the dominant emittance growth mechanism for this situation would be the effect of from radial velocities in the beam leading to a change in the particles' energies. The rms divergence is about $10 \mathrm{mrad}$ for this case, and the emittance growth is about $20 \mathrm{~mm} \mathrm{mrad}$ per meter of the focusing channel (after we remove the $20 \mathrm{~mm}$ mrad per meter emittance growth from the space-charge force), in agreement with the estimate in Section III.C, based on the divergence term in Eqn. (29).

In Fig. 7, we examine the case the scalloping is due to a mismatch in a uniform focusing channel. The radial profile is plotted in Fig. 7a, and the emittance evolution is shown in Fig. 7b. Using Eqn. (66), we would suspect that the emittance growth for this beam profile (an rms divergence of about $10 \mathrm{mrad}$ ) would be about $15 \mathrm{~mm}$ mrad per meter of oscillation, less than half of that actually seen in Fig. $7 \mathrm{~b}$. The majority of the emittance growth in this case is actually due to the variation in the radial space-charge force and diamagnetic field from density nonuniformities as the beam is propagated. By modifying SLICE so that the radial velocity term is ignored, the emittance growth from the space-charge and diamagnetic effects is about $30 \mathrm{~mm}$ mrad per meter of drift (see Fig. 7c). The effect of the beam's radial divergence is then about $20 \mathrm{~mm}$ mrad per meter of transport (assuming that the effects are uncorrelated) which is close to that predicted.

\section{Integrated Test Stand Example}

In this section we consider the emittance growth in an actual beamline with solenoid focusing. The Integrated Test Stand (ITS) is a $6-\mathrm{MeV}$ induction linearaccelerator with a $3.75-\mathrm{MeV}$ injector. In Fig. 8a we show the beam radius profile for the nominal machine tune, with a final focus at about $9.5 \mathrm{~m}$. The simulation starts at an axial position of $18.1 \mathrm{~cm}$, at the location of the injector anode. In Fig. $8 \mathrm{~b}$ the average energy is plotted as a function of axial position. The $200-\mathrm{keV}$ induction accelerating cell locations are clear from this plot. The slight decreases in the average energy is due to the conversion of beam kinetic to potential energy as the beam is radially compressed. In Fig. $8 \mathrm{c}$ the profile of the axial magnetic field is plotted, showing the locations and 
strengths of the various solenoids. The emittance evolution is shown in Fig. 8d (for an initially low emittance beam), and the final transverse configuration and phase space distributions are shown in Fig. 8e. Using the earlier examples, we can identify the main emittance growth mechanisms along the beamline: (1) After the anode magnet, there is a substantial emittance growth due to the strong convergence of the beam. (2) The emittance decreases as the beam is focused at the start of the transport channel. (3) The emittance oscillates and grows slightly in the transport channel due to oscillations in the beam size (the emittance growth is from the radial divergence of the beam). (4) A very large emittance growth occurs in the region between the third and second to last solenoids, where the radial divergence is the largest. (5) Some additional emittance growth occurs in the second-to-last solenoid from the variation in axial velocities. (6) In the drift to the final focus, the emittance growth from the beam convergence is countered by the tendency of the beam's correlations to be compensated in the focus; there is a sudden drop in the emittance at the location of the final focus.

The actual ITS injector has an initial emittance of about $1000 \mathrm{~mm}$ mrad. Using the initial transverse configuration and phase space distributions shown in Fig. 8f, we can simulate the emittance evolution in the ITS injector starting with this initial emittance. The emittance evolution for this case is shown in Fig. 8g, and the emittance growth is hidden by the initial emittance.

\section{E. Nonuniform Beam Density}

Using SLICE, we can evaluate the emittance growth for a beam with an initially nonuniform transverse density, with uniform balanced flow. The initial transverse distribution for a quadratic distribution is shown in Fig. 9a. The radial beam profile is plotted in Fig. $9 \mathrm{~b}$ and the emittance growth is plotted in Fig. 9c. As we have seen several times before, the emittance oscillates as radial correlations are created and eliminated; however, the emittance growth is about 20 times larger than before, at about $500 \mathrm{~mm}$ mrad per meter. 


\section{Discussion}

In the previous sections, we have considered quite a few different emittance growth mechanisms for various focusing scenarios, using either solenoids or quadrupoles. In this section, we will summarize the major emittance growth mechanisms, which ones accumulate along the beamline, and make other observations in order to lead to some useful insights about the problem. First, in the table below, we summarize the earlier results for the ITS parameters:

\begin{tabular}{l|c|c|c} 
& $\begin{array}{c}\text { Solenoid } \\
\text { focusing }\end{array}$ & $\begin{array}{c}\text { Quadrupole } \\
\text { focusing }\end{array}$ & \multicolumn{1}{|c}{$\begin{array}{c}\text { Emittance growth } \\
\text { mechanisms }\end{array}$} \\
\hline $\begin{array}{l}\text { Uniform balanced } \\
\text { flow }\end{array}$ & $20 \pi \mathrm{mm} \mathrm{mrad} / \mathrm{m}$ & $<1 \pi \mathrm{mm} \mathrm{mrad} / \mathrm{m}$ & $\begin{array}{l}\text { - nonlinearity in space- } \\
\text { charge force } \\
\text { - variation of axial } \\
\text { velocities in the space- } \\
\text { charge force }\end{array}$ \\
\hline Final focus & $100 \pi \mathrm{mm} \mathrm{mrad}$ & $50 \pi \mathrm{mm} \mathrm{mrad}$ & $\begin{array}{l}\text { - variation of axial } \\
\text { velocities and radial } \\
\text { vector potential } \\
\text { - cancellation of radial } \\
\text { vector potential with } \\
\text { potential depression } \\
\text { - divergence term } r_{o}^{\prime 2}\end{array}$ \\
\hline Scalloped flow & $15 \pi \mathrm{mm} \mathrm{mrad/m}$ & $15 \pi \mathrm{mm} \mathrm{mrad} / \mathrm{m}$ & - divergence term $r_{o}^{\prime 2}$
\end{tabular}

Note that the emittance growth from the space-charge force scales as $1 / \gamma$ and will vanish for sufficiently high beam energies. The emittance growths from the radial vector potential, the potential depression (for the emittance growth in a quadrupole focusing field), and the divergence term all are independent of $\gamma$. However, the emittance growth from the variation of the axial velocity in a solenoidal field scales linearly with $\gamma$ (this is because we are assuming the focal length is kept constant); this is a rather unfortunate scaling, but both the minimum spot size and even emittance growth in the solenoid will still decrease with increasing energy because of the scaling of the beam size in the solenoid (Eqn. (49)). The unnormalized emittance decreases as $1 / \gamma$ and 
as a result the beam size in the solenoid will decrease, and there will be consequently less emittance growth.

The emittance growth from the space-charge force nonlinearity (for the solenoid case) is independent of beam radius, as is the emittance growth from the divergence of a scalloping beam. The emittance growth from the radial vector potential scales as the square of the beam radius, as does the emittance growth from the potential depression in the quadrupole focusing field and the emittance growth from the beam divergence in the final focus.

The emittance growth from the vector potential term is not cumulative along the beamline - the only thing that matters is the final convergence angle of the beam. It is easy to see from Eqn. (34) that the contributions from inward radial acceleration (from the focusing element) will cancel the contributions from outward radial acceleration (from the space-charge force). However, the contributions from the divergence terms will add cumulatively throughout the beamline over a correlation length. Likewise for solenoidal focusing, the contributions from the variation in the axial velocity will only occur when the beam is focused in a solenoid, and these contributions will also add cumulatively. This is also true for the emittance growth from any nonlinearities in the space-charge force.

The three most important emittance growth mechanisms are (1) current density nonuniformities, (2) beam scalloping in a mismatched magnetic field, and (3) beam divergence/convergence effects. These effects will dominate the emittance growth; the first two effects can be minimized by proper design and operation of the machine. However, some beam current density nonuniformity always occurs (see for example Fig. $8 \mathrm{e}$ ), and this effect, along with unavoidable beam radius fluctuations (including convergence to a final target), will lead to a minimum possible emittance growth.

\section{References}

1. Y.-J. Chen, "Corkscrew modes in linear accelerators," Nucl. Instrum. and Meth. Phys. Res., A292, $455-464$ (1990). 
2. W. C. Turner, et al., "Reduction of beam corkscrew motion on the ETA-II linear induction accelerator," Proc. 1990 Linear Accel. Conf., 435-437, (Albuquerque, NM, Sept. 10-14, 1990).

3. Y.-J. Chen, "Beam control in the ETA-II linear induction accelerator," Proc. 1993 Part. Accel. Conf., May 1993).

4. M. Burns, P. Allison, J. Downing, D. Moir, G. Caporaso, and Y. Chen, "Technology demonstration for the DARHT linear induction accelerator," Proc. 9th Intern. Conf. on High Power Particle Beams, Washington, DC, May 25-29, 1992.

5. M. Reiser, "Laminar-flow equilibria and limiting currents in magnetically focused relativistic beams," Phys. of Fluids, 20, 477-486 (1977).

6. J. F. Gittens, Power travelling-wave tubes, American Elsevier Publishing Company, Inc., New York, 1965.

7. P. Lorrain and D. R. Corson, Electromagnetic fields and waves, W. H. Freeman and Company, San Francisco, 1970.

8. P. Allison, "DARHT performance prediction for lower emittance,". DARHT Tech. Note 42 (1993).

9. E. Lee, "Cancellation of the centrifugal space-charge force," Particle Accelerators, 25, $241(1990)$.

10. B. E. Carlsten and T. O. Raubenheimer, "Emittance growth of bunched beams in bends," Phys. Rev. E, 51, 1453-1470 (1995). 
11. B. E. Carlsten, "Space-charge-induced emittance compensationin high-brightness photoinjectors," Particle Accelerators, 49, 27 (1995). 


\section{Figures}

1. Radial vector potential at the beam edge (normalized to the beam potential energy depression) versus axial length of integration, for an edge divergence of 0.01 radians.

2. Radial vector potential (normalized to the beam potential energy depression) versus radial position within the beam, for an edge divergence of 0.01 radians.

3. Radial vector potential at the beam edge (normalized to the beam potential energy depression) versus edge divergence.

4. Numerical results for the balanced flow case. (a) Radial beam profile. (b) Emittance profile.

5. Numerical results for the hard focusing case. (a) Radial beam profile. (b) Applied axial field profile (not including beam diamagnetic effects). (c) Average beam energy profile. (d) Emittance profile. (e) Final beam transverse phase space and configuration space. (f) Emittance profile in the absence of the emittance growth from the beam radial divergence.

6. Numerical results for the beam scalloping with periodic focusing case. (a) Radial beam profile. (b) Applied axial field profile (not including beam diamagnetic effects). (c) Emittance profile.

7. Numerical results for the beam scalloping in a constant axial magnetic field case. (a) Radial beam profile. (b) Emittance profile. (c) Emittance profile in the absence of the emittance growth from the beam radial divergence. 
8. Integrated Test Stand case. (a) Radial beam profile. (b) Average beam energy profile. (c) Applied axial field profile at the center of the beam (not including beam diamagnetic effects). (d) Emittance profile starting with a very low initial emittance. (e) Initial beam transverse phase space and configuration space for an initial emittance of $1000 \mathrm{~mm} \mathrm{mrad}$. (f) Emittance profile starting with an emittance of $1000 \mathrm{~mm}$ mrad.

9. Nonuniform beam density case. (a) Initial radial current-density profile. (b) Radial beam profile. (c) Emittance profile. 


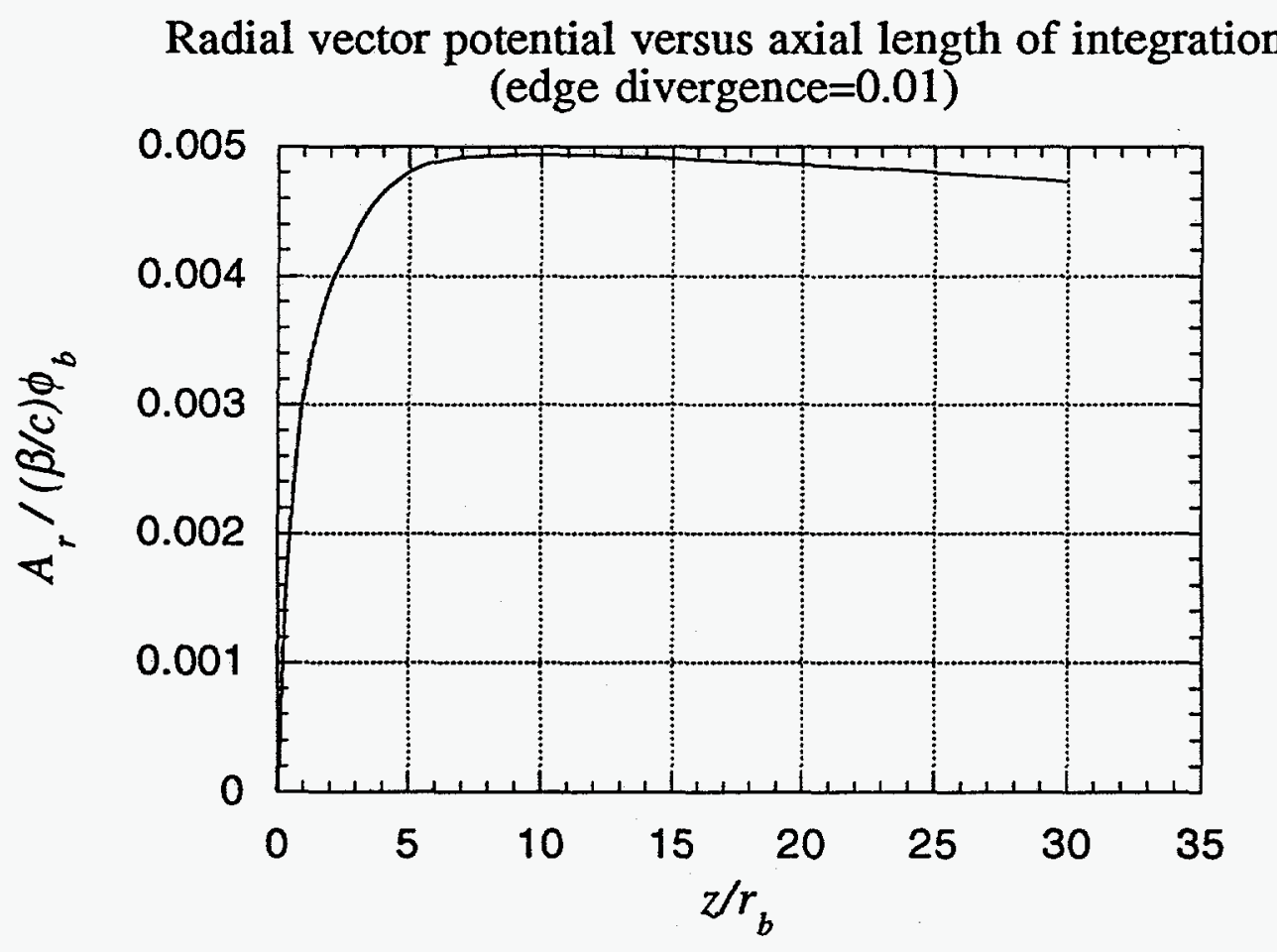

Fy 1 
Radial vector potential versus radial position in beam (edge divergence $=0.01$ )

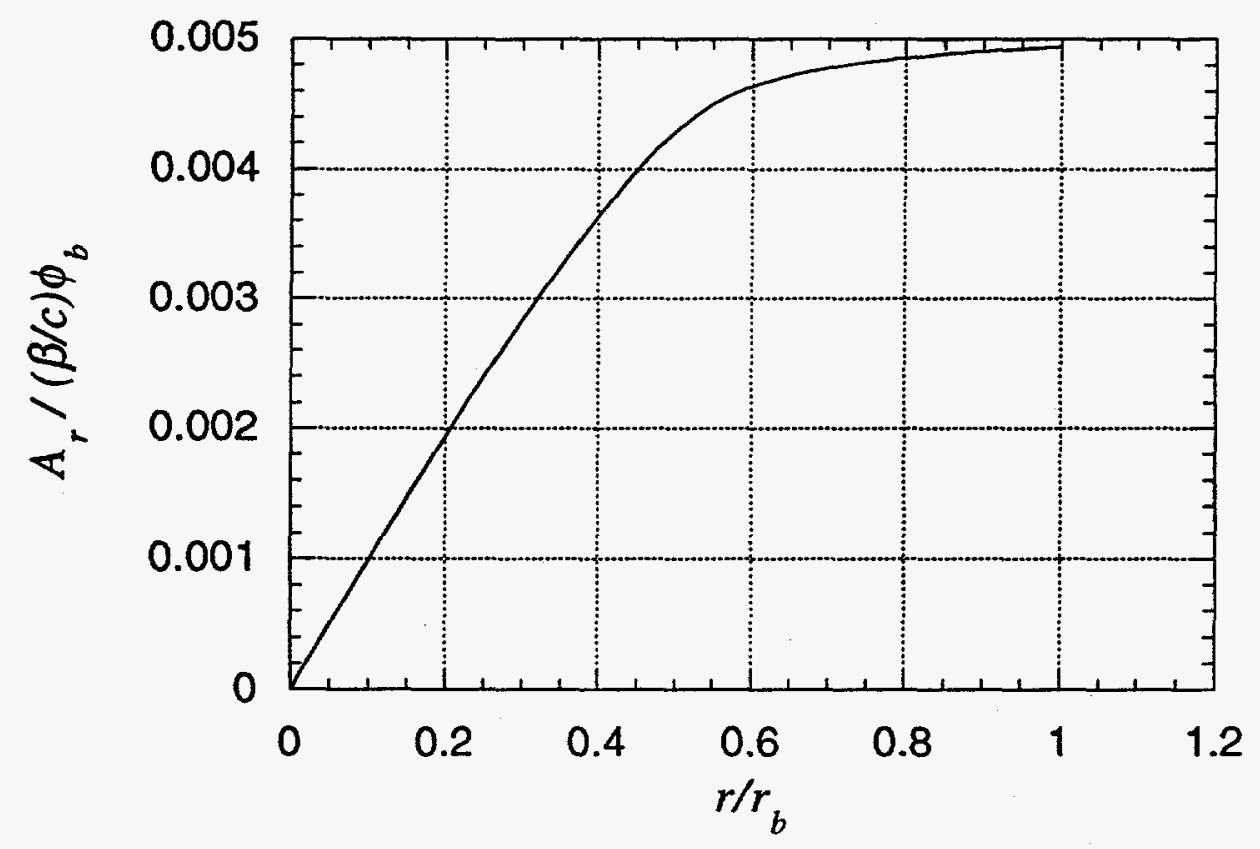

$\operatorname{rg} 2$ 
Radial vector potential versus edge divergence

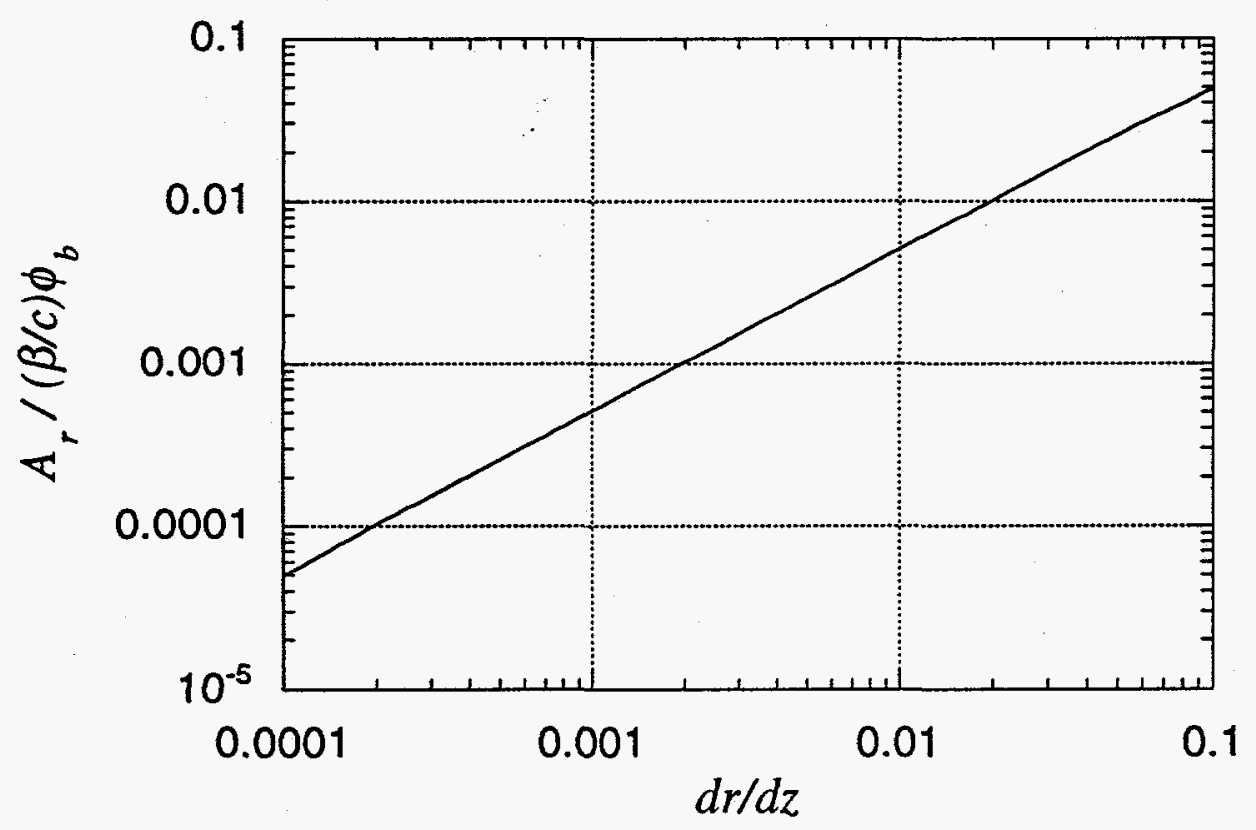

Figs 


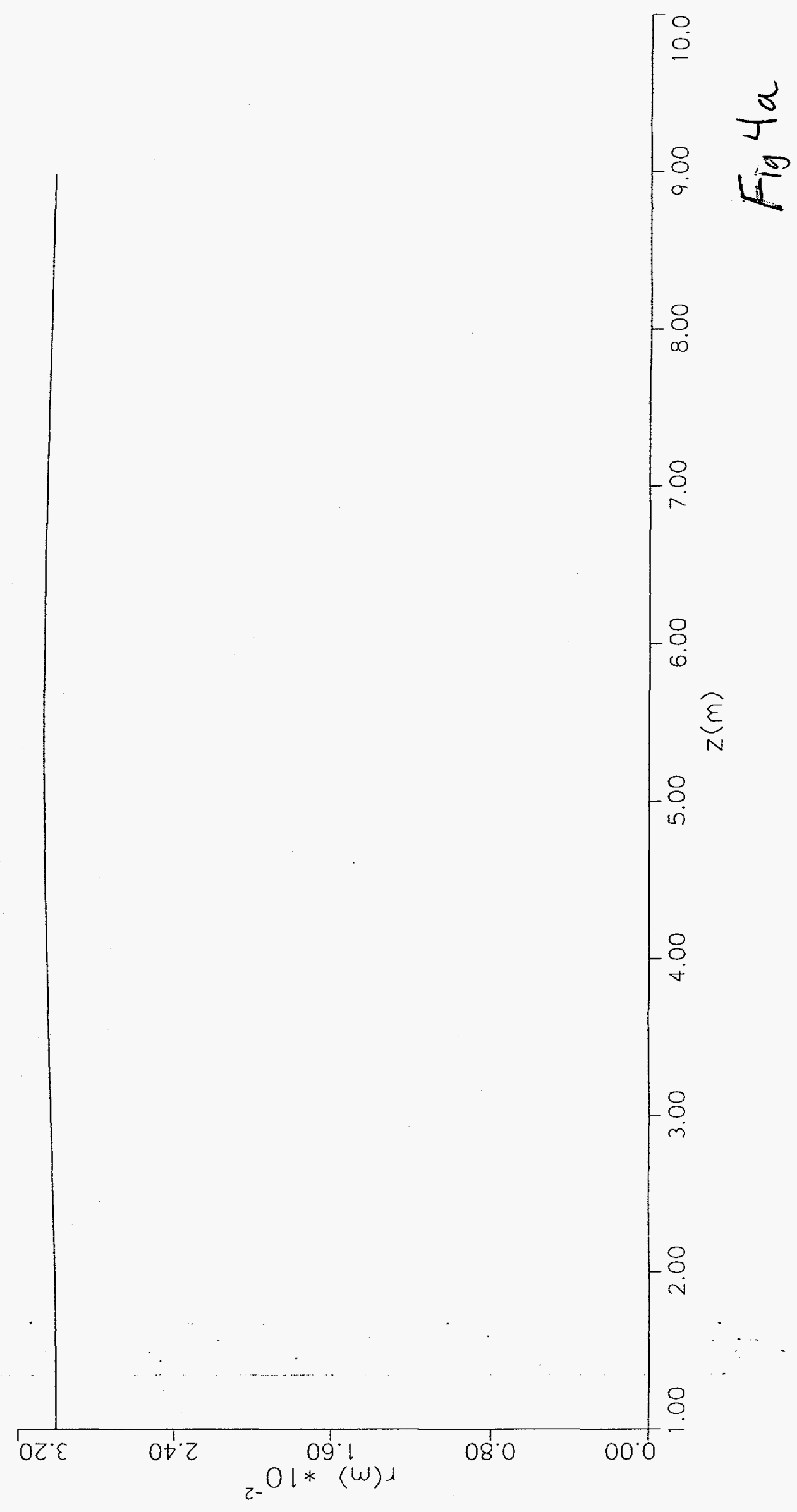




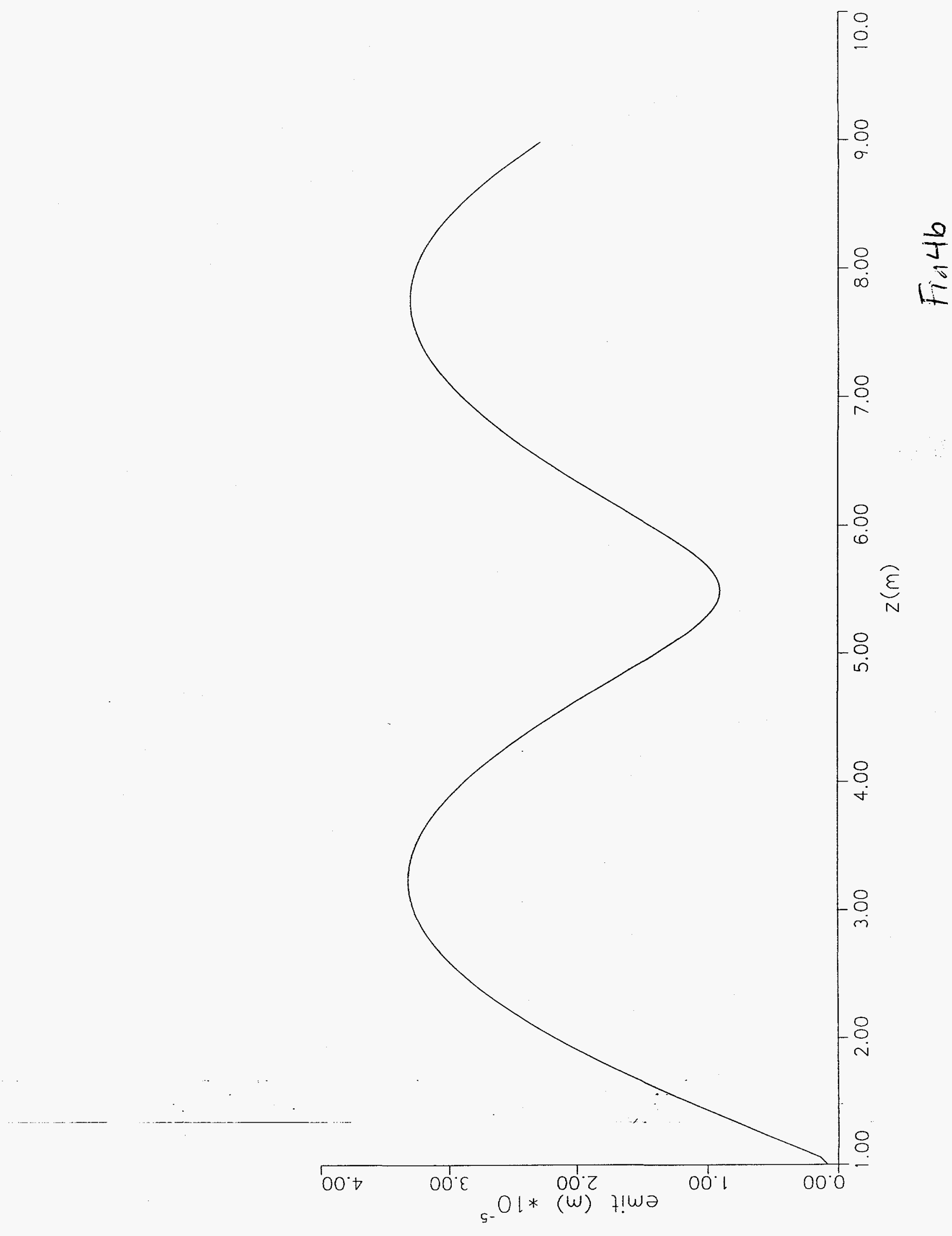




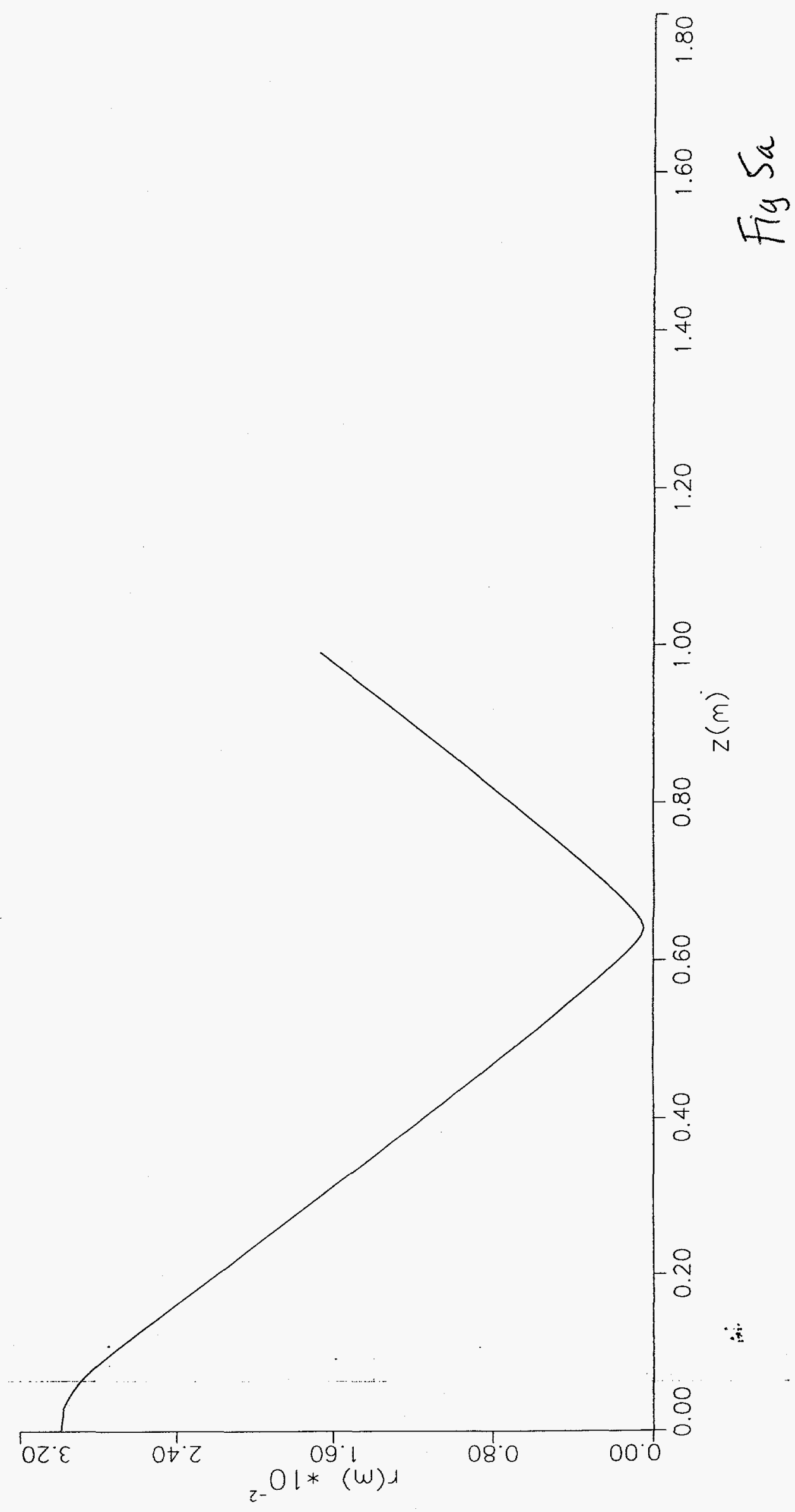




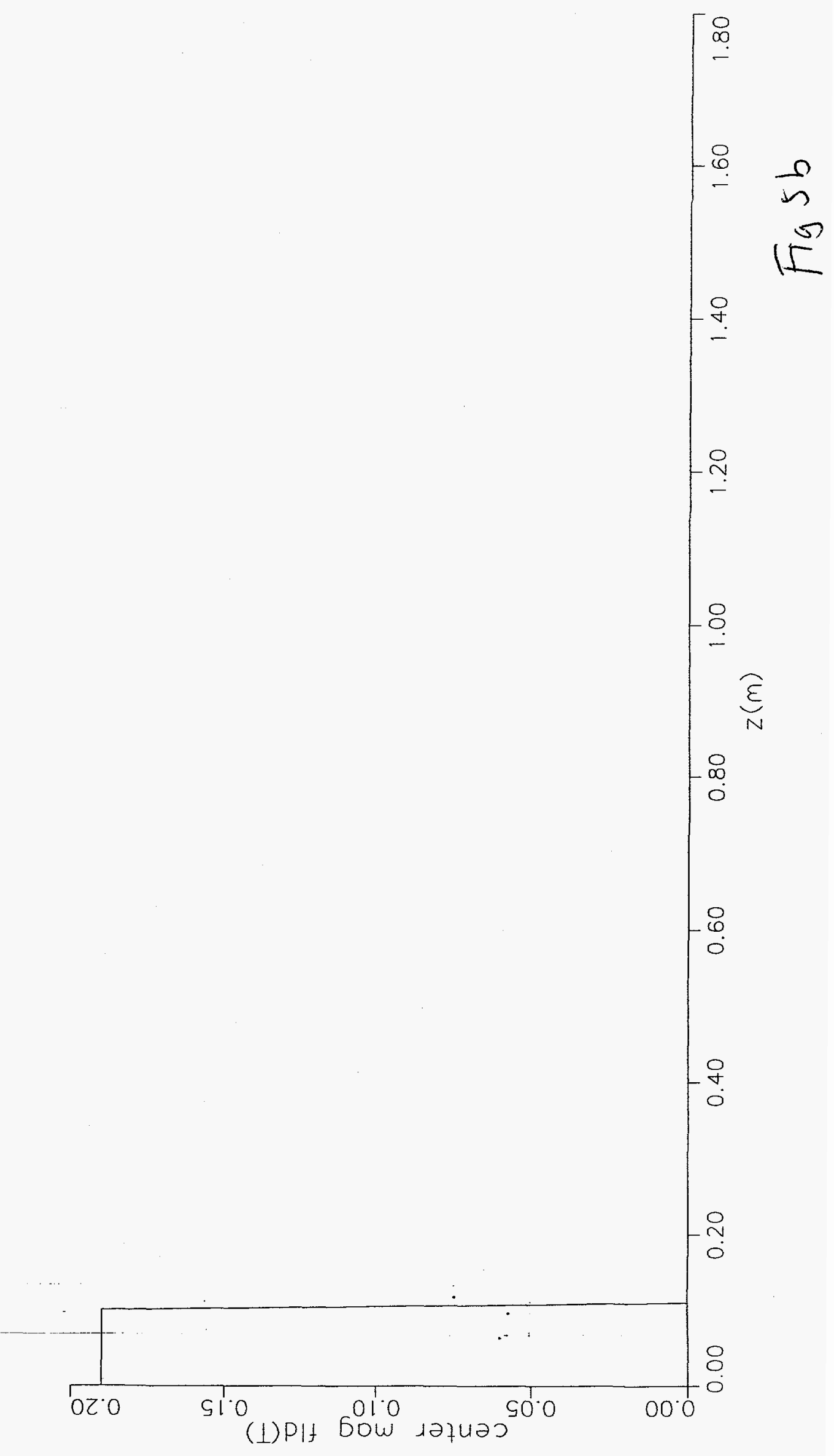




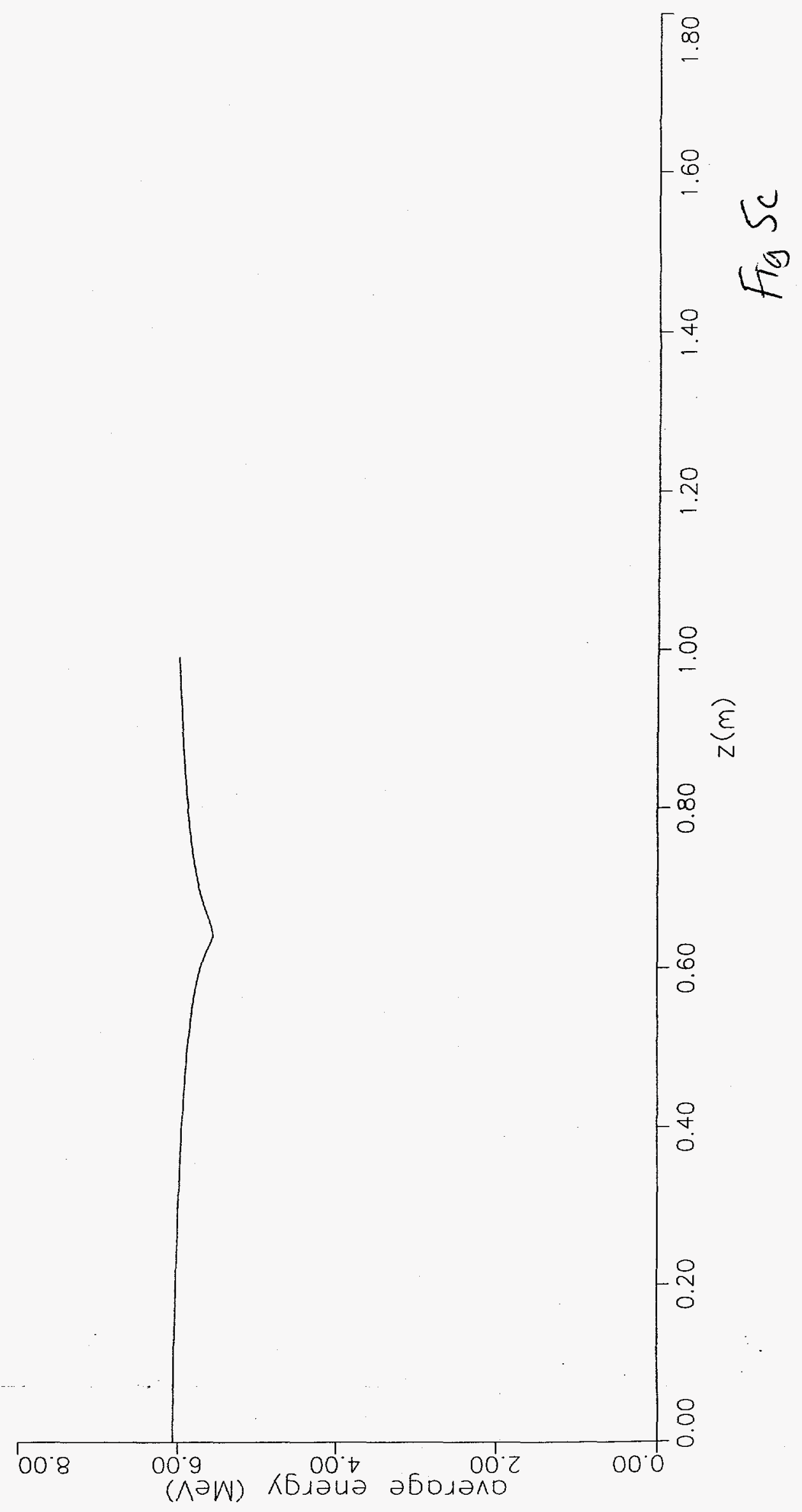




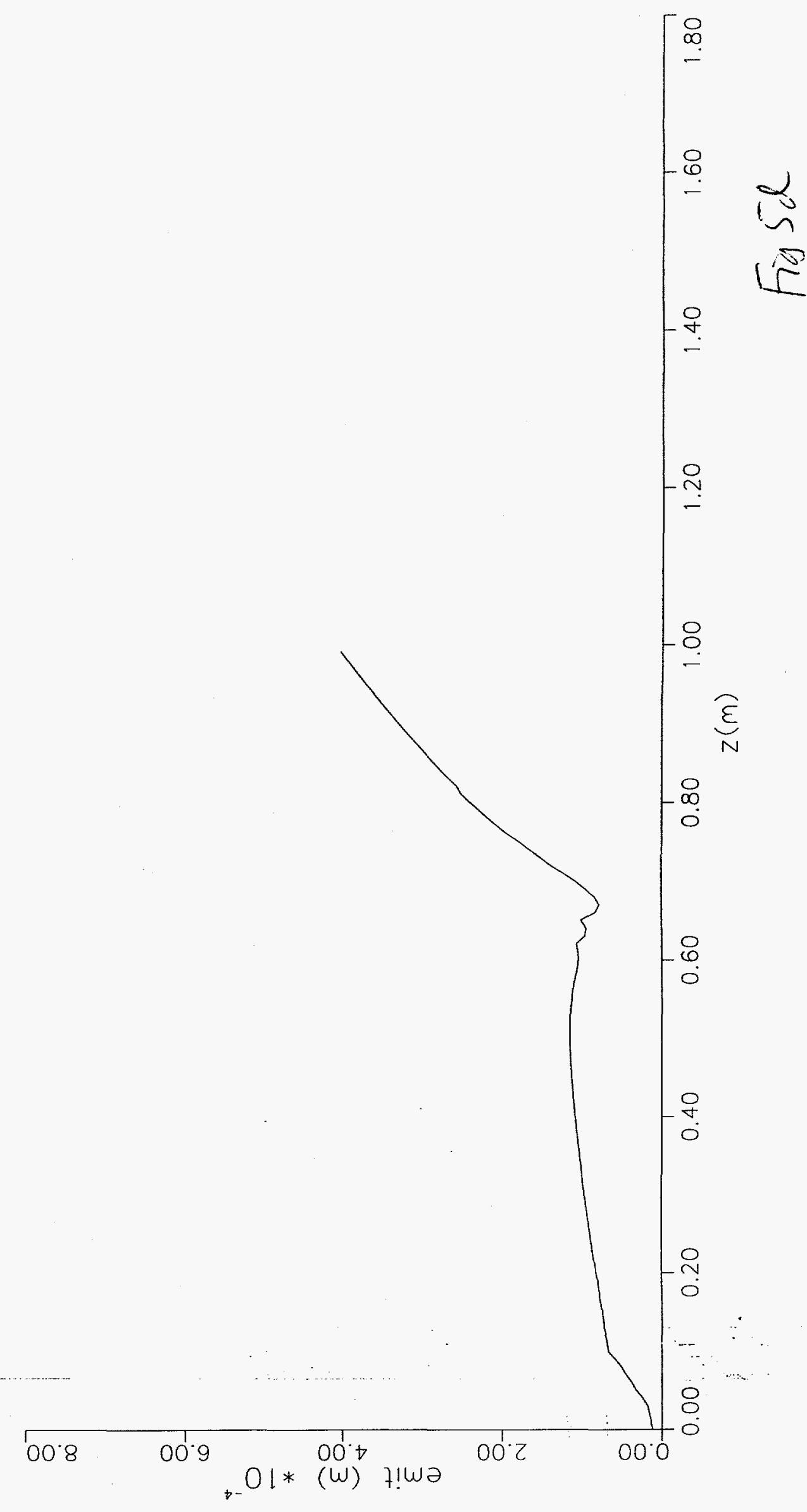



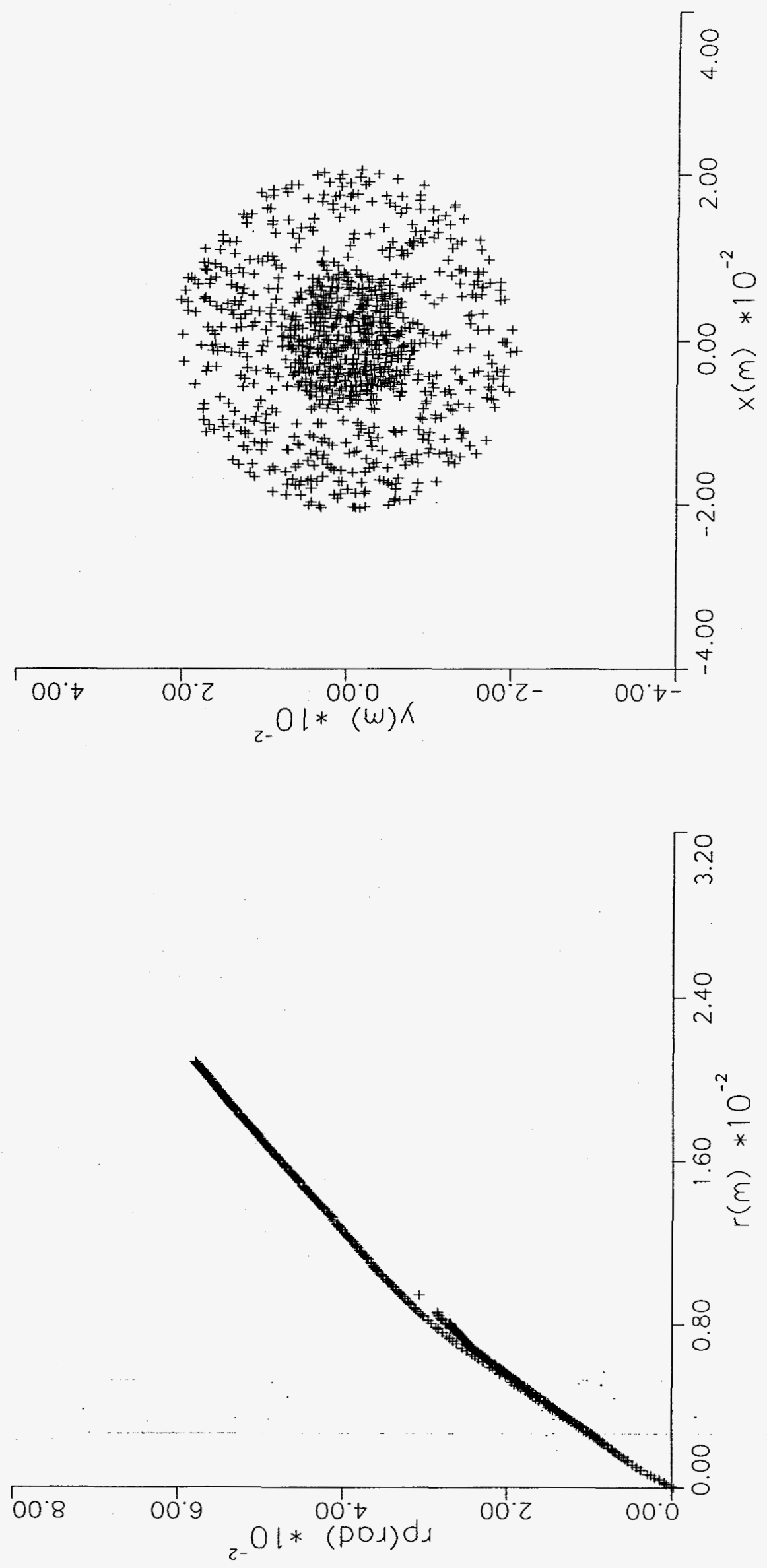


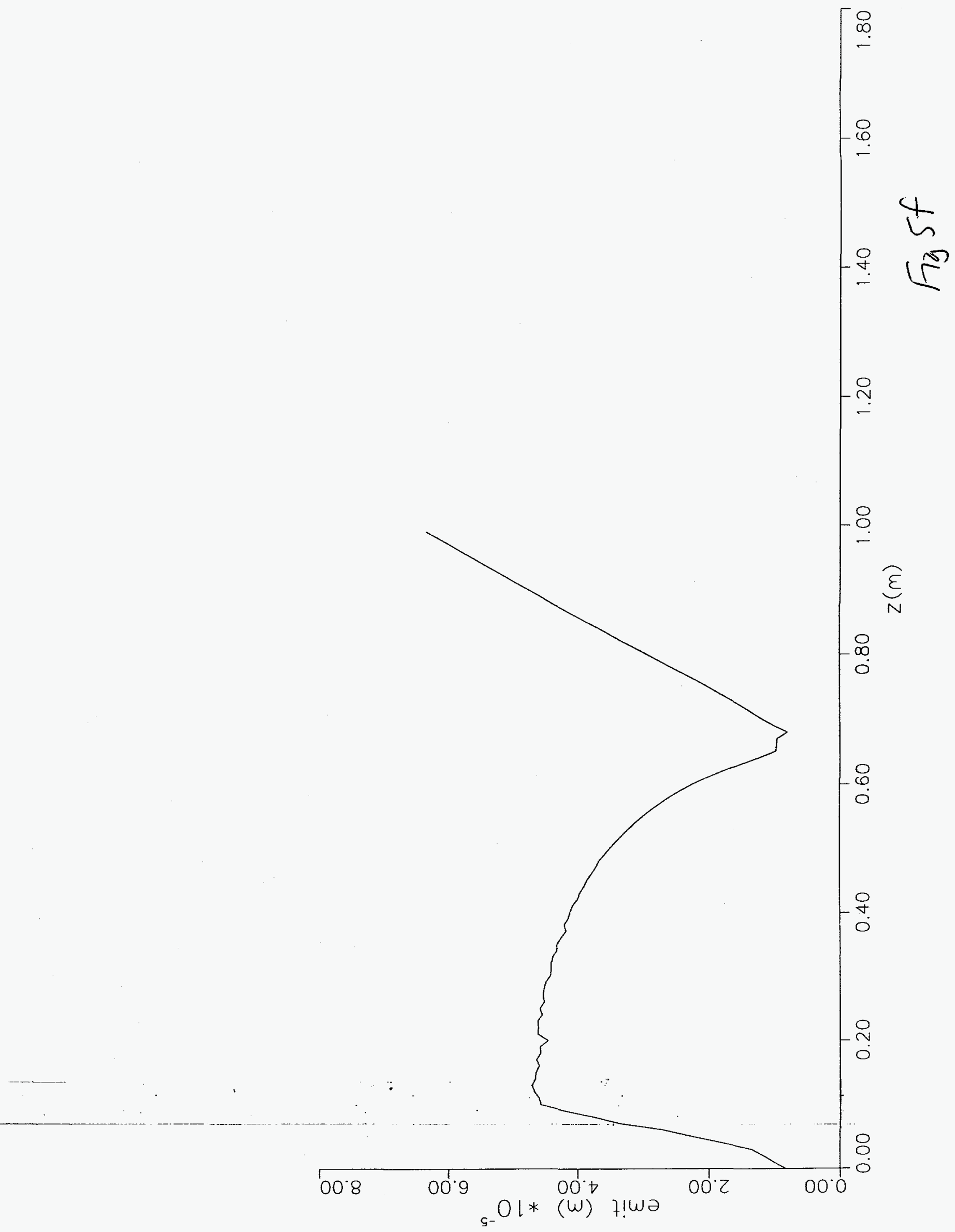




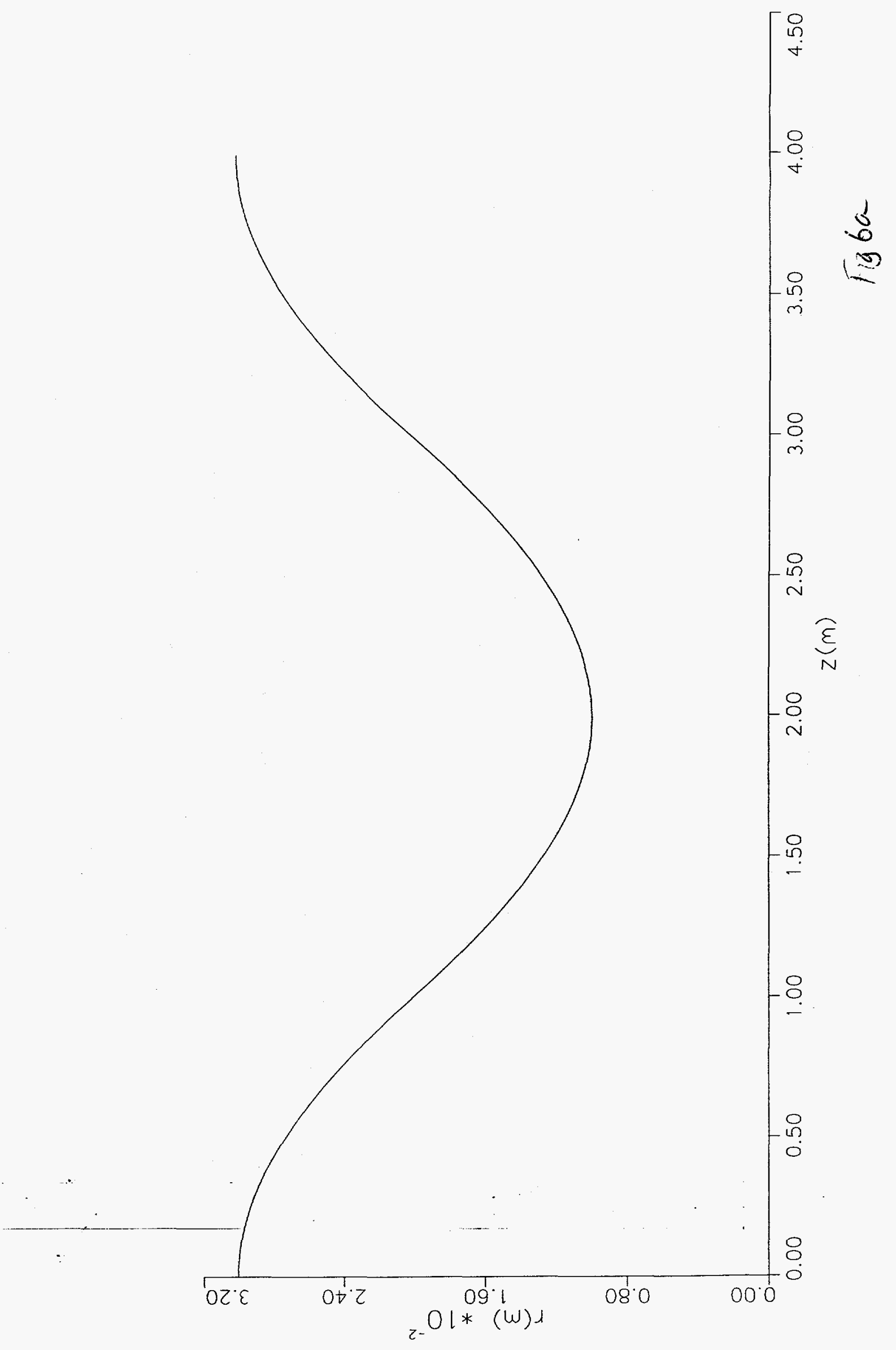




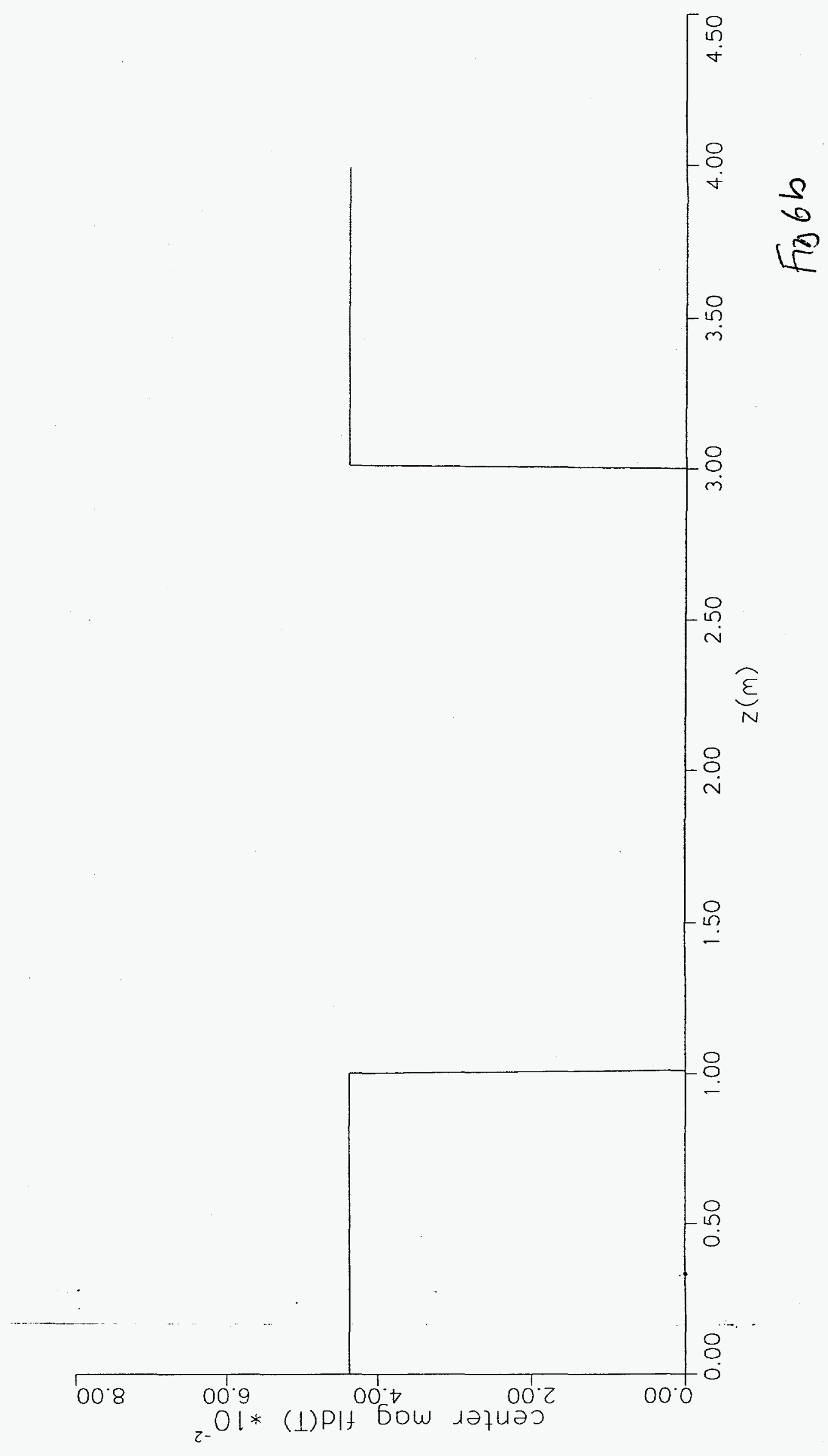




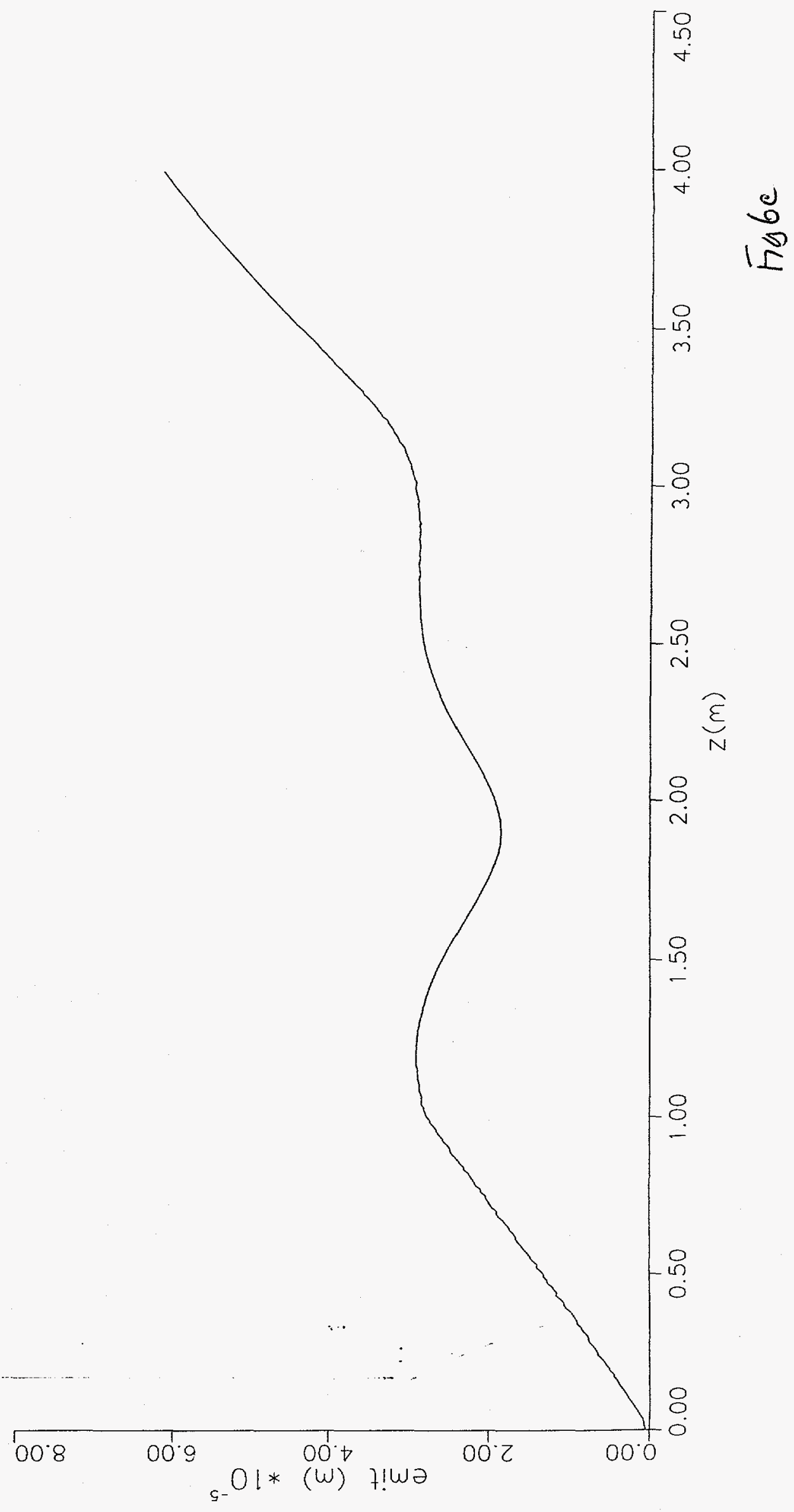




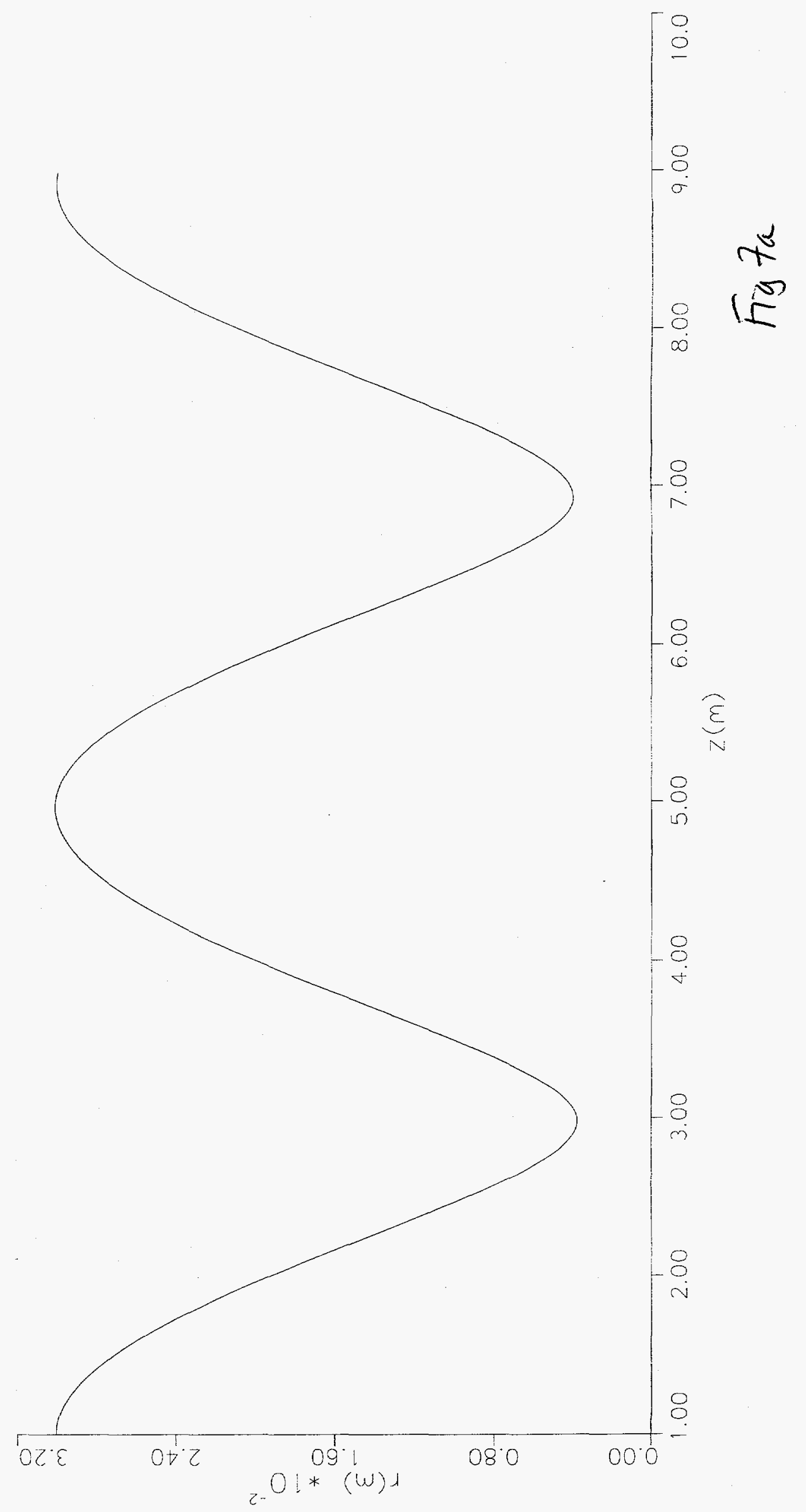




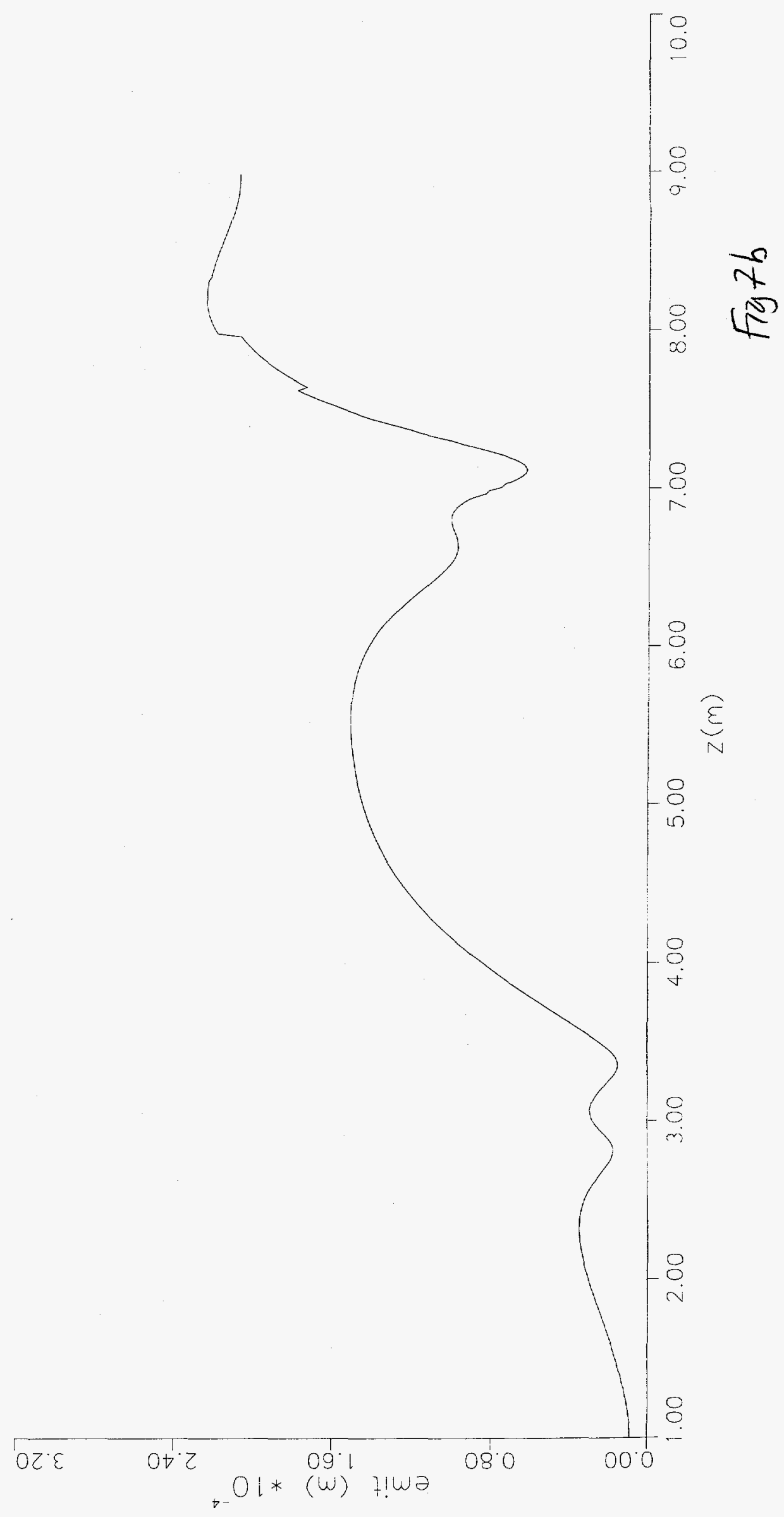




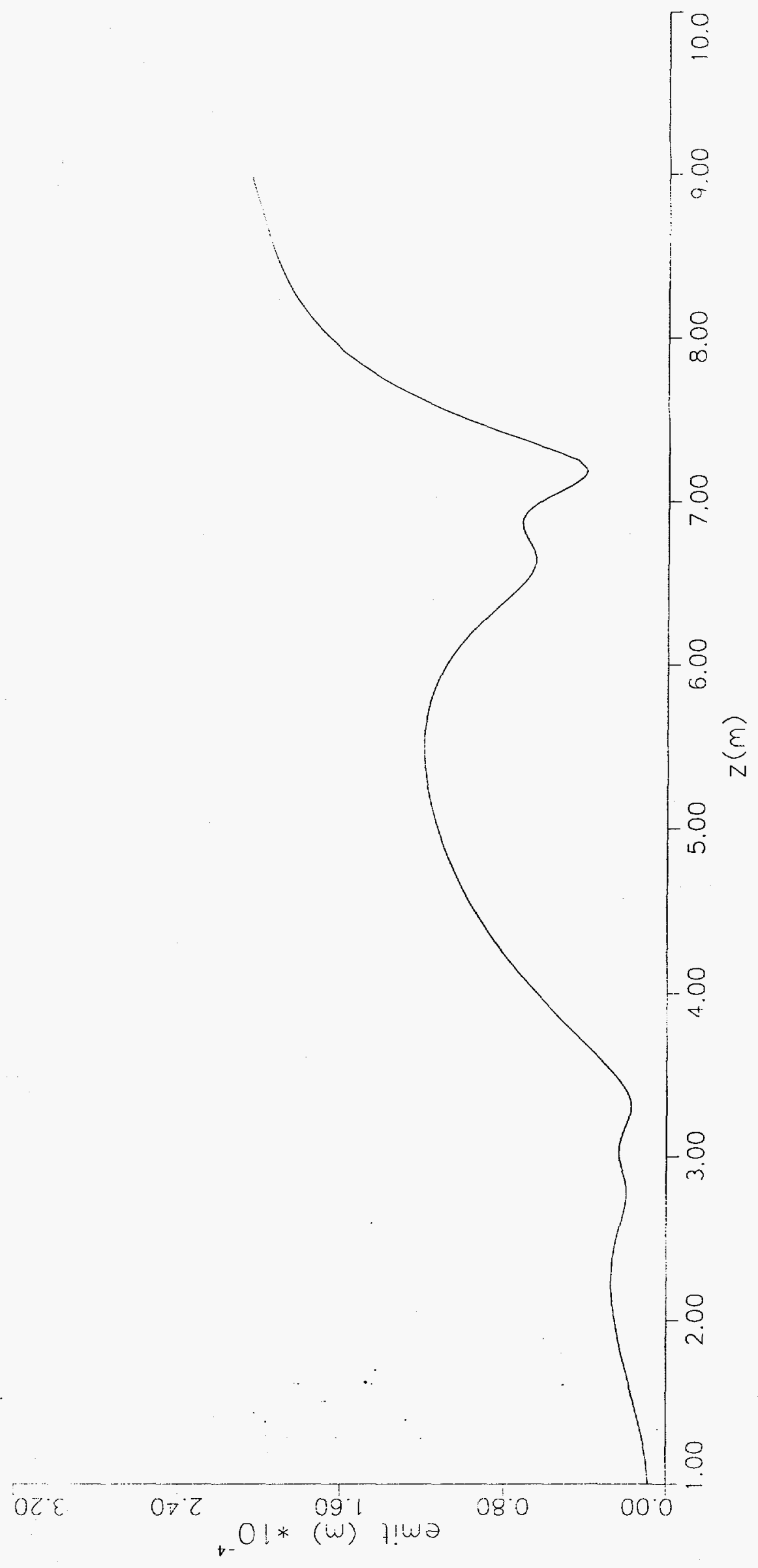




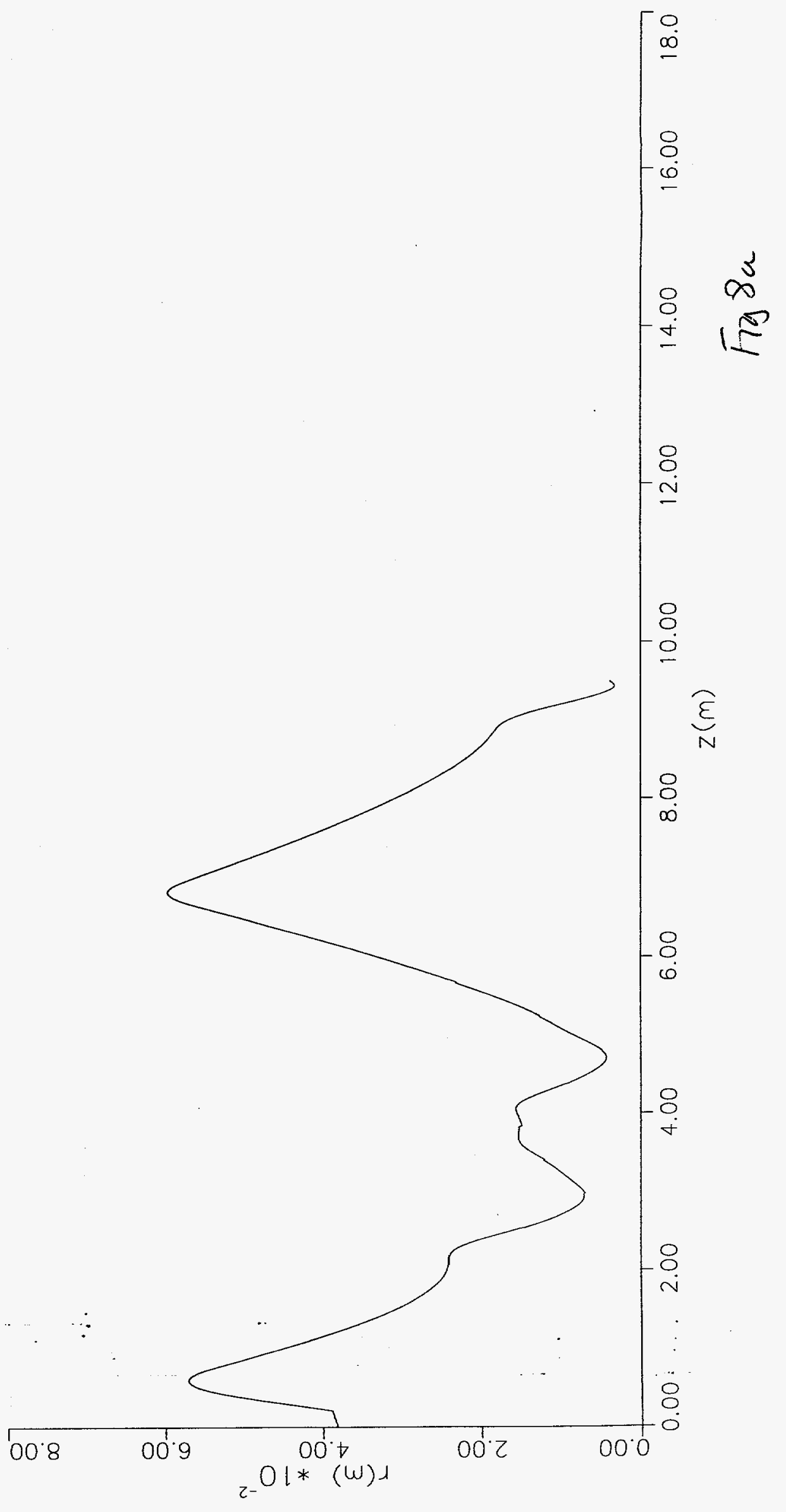





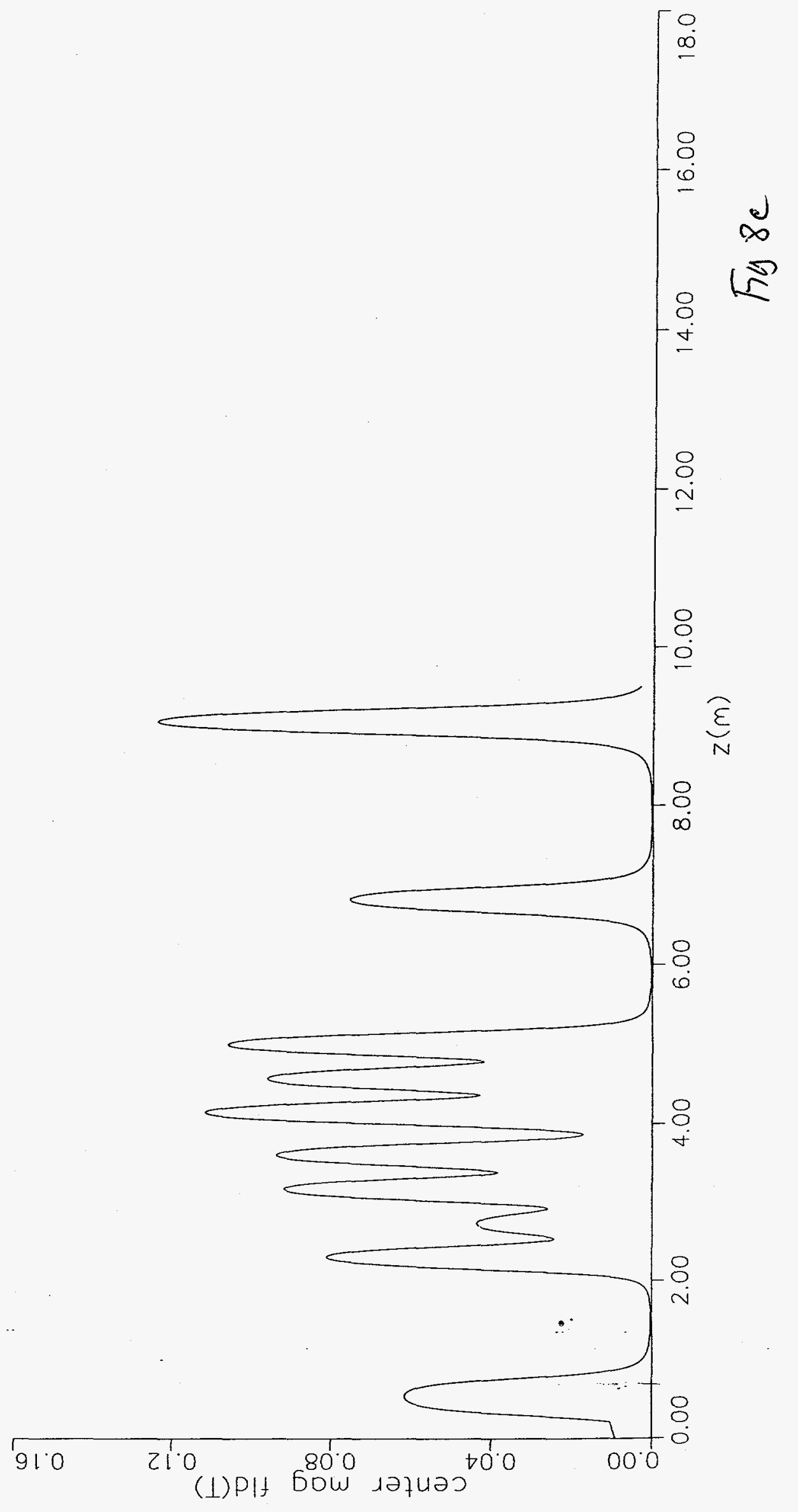




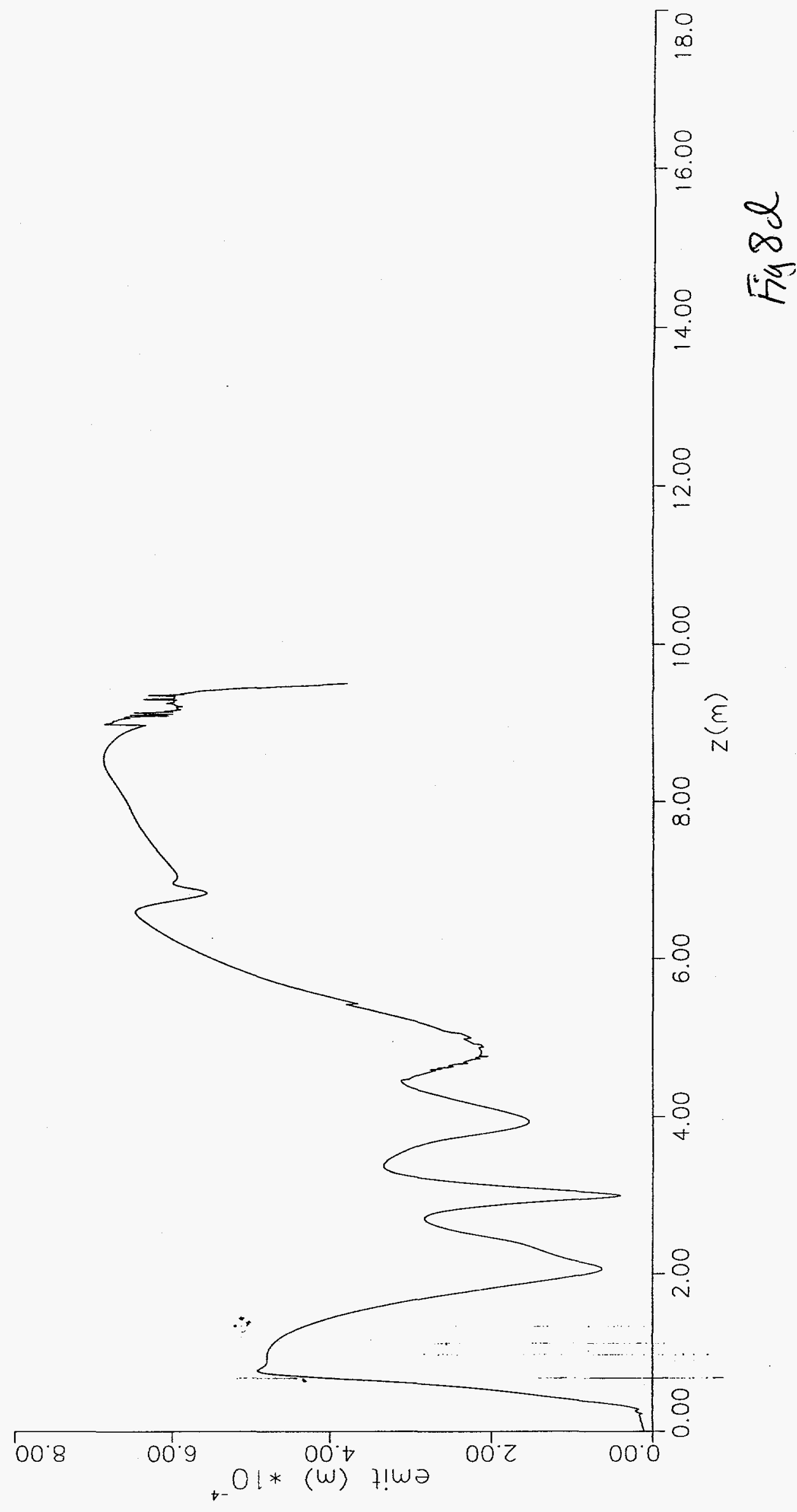




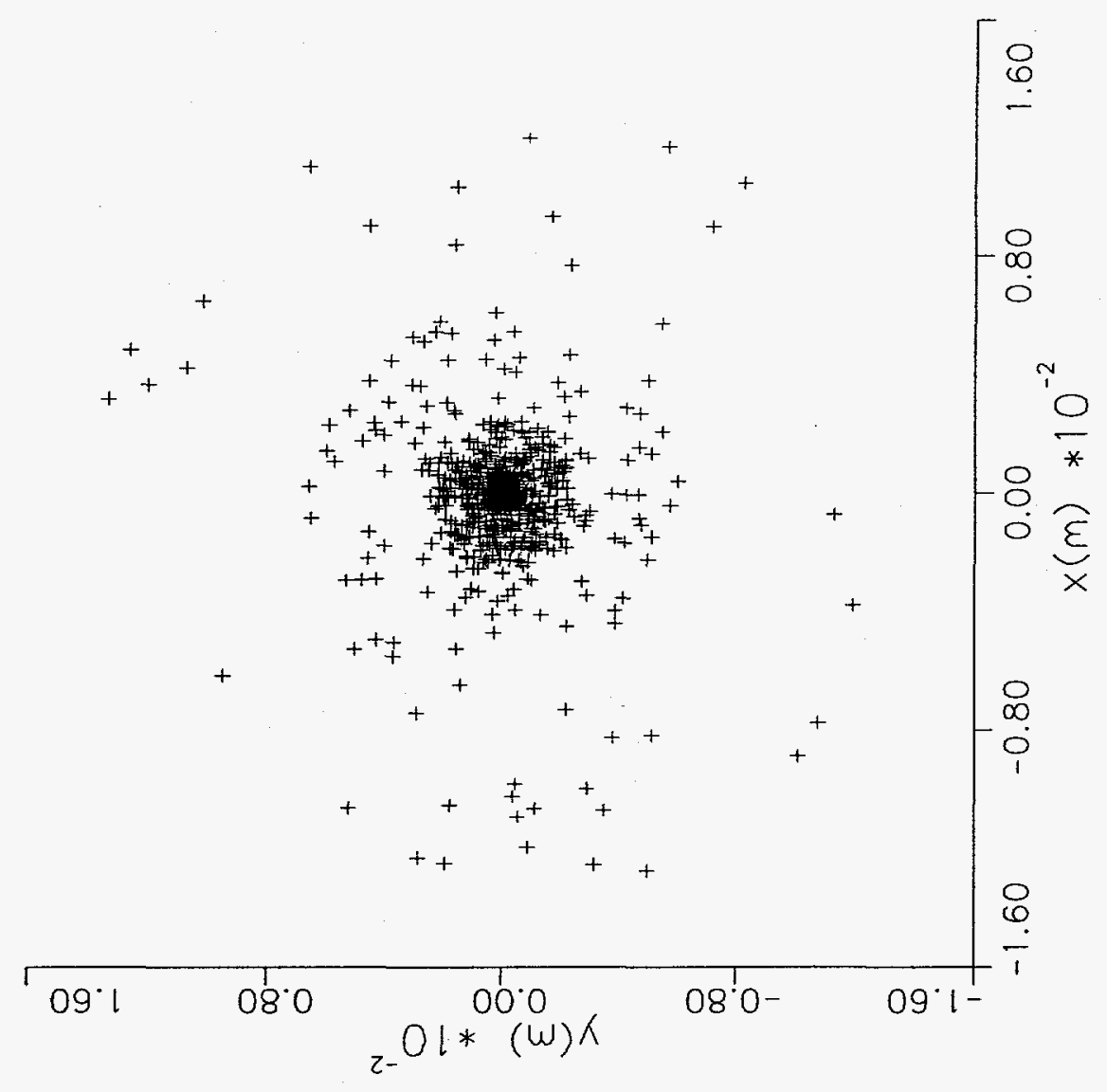

0
0
1

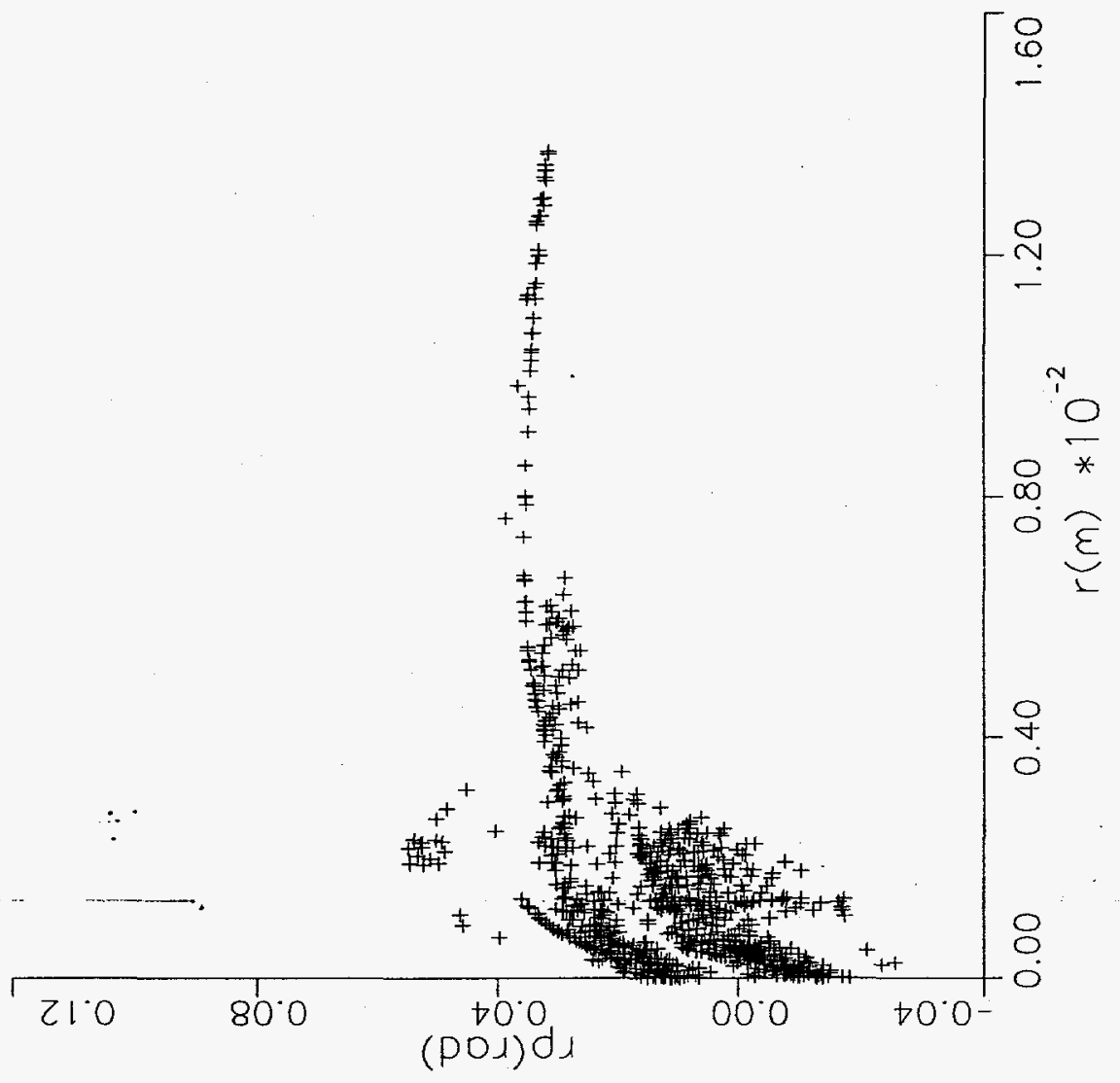




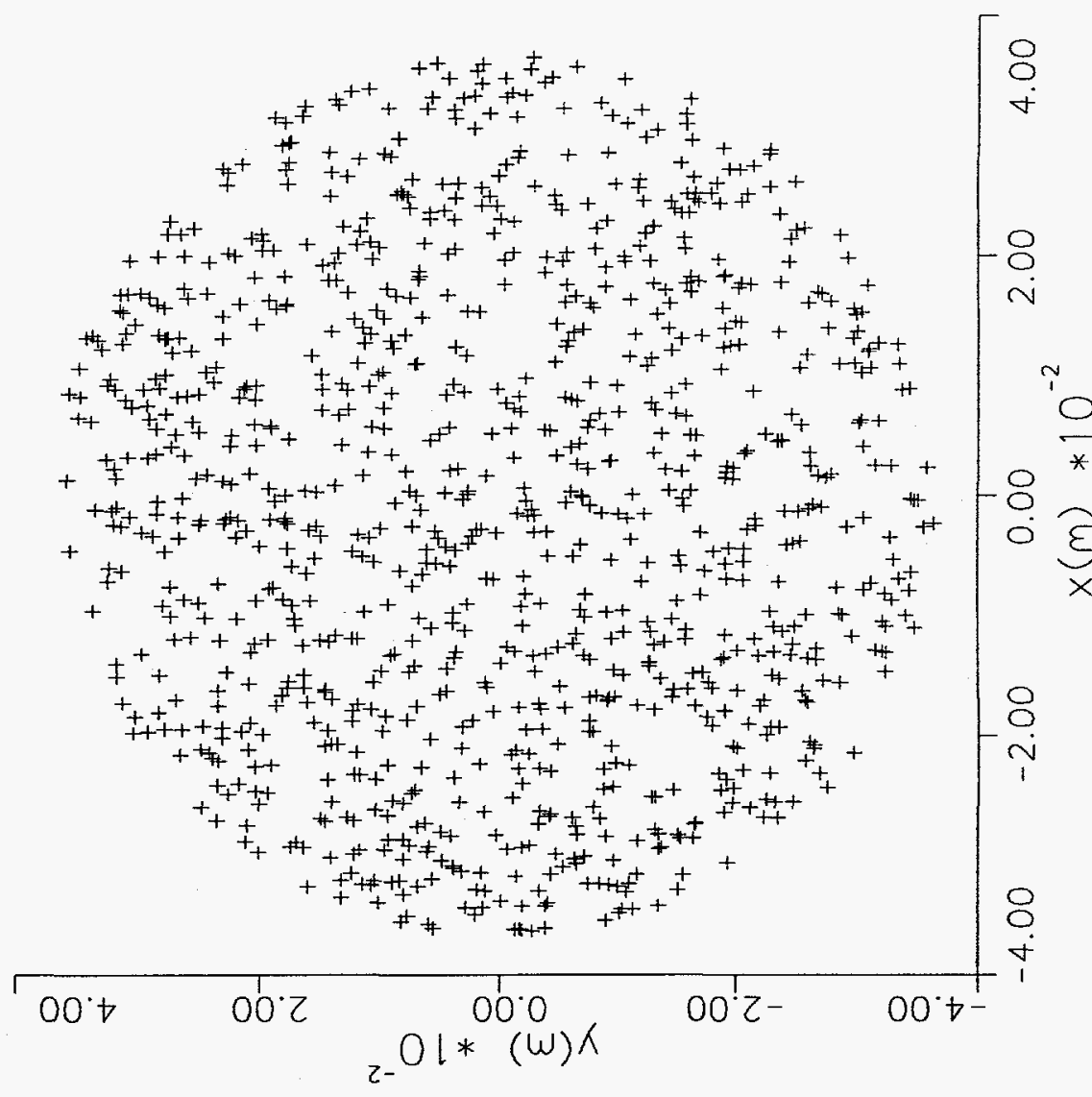

4

N

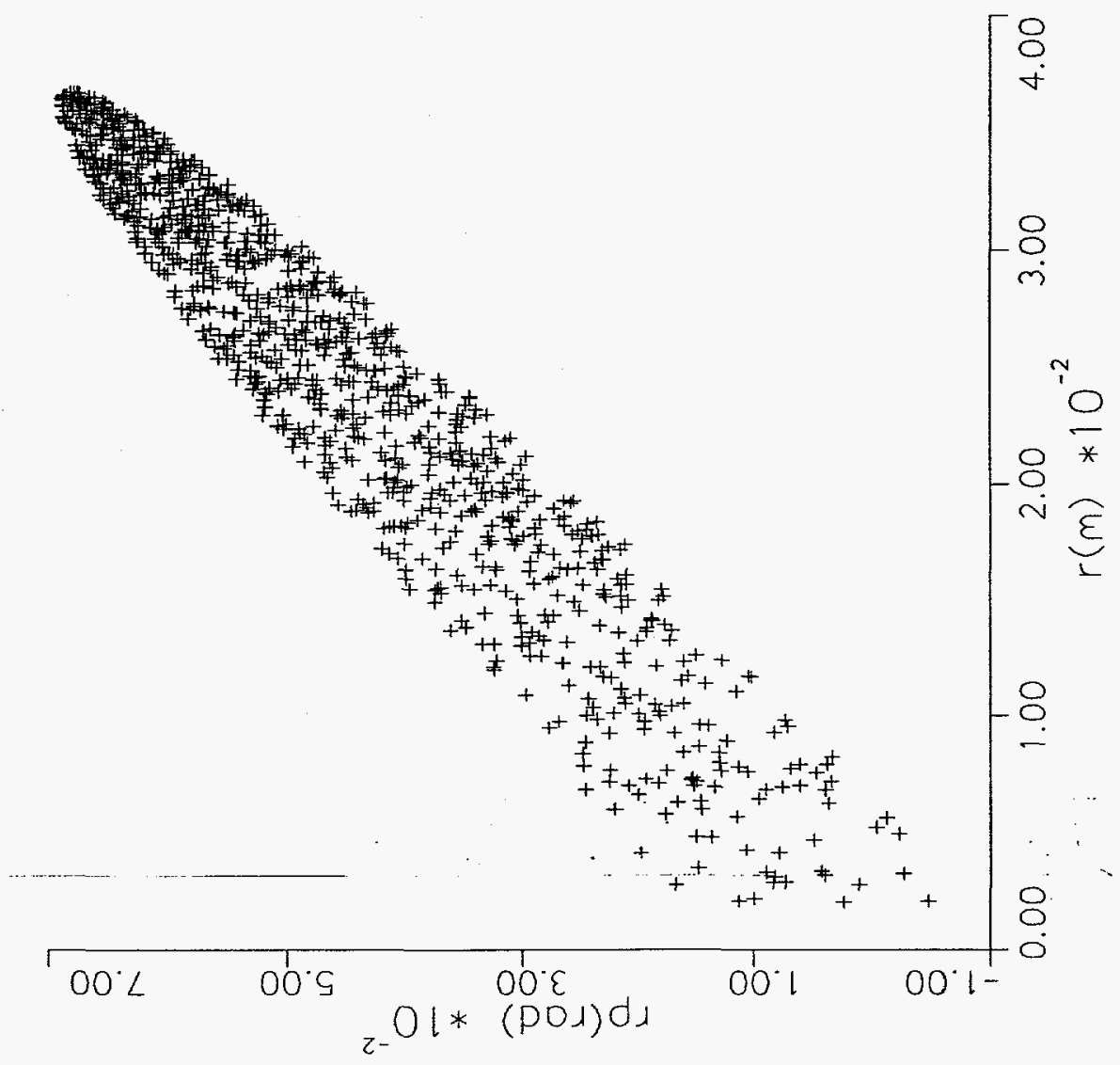





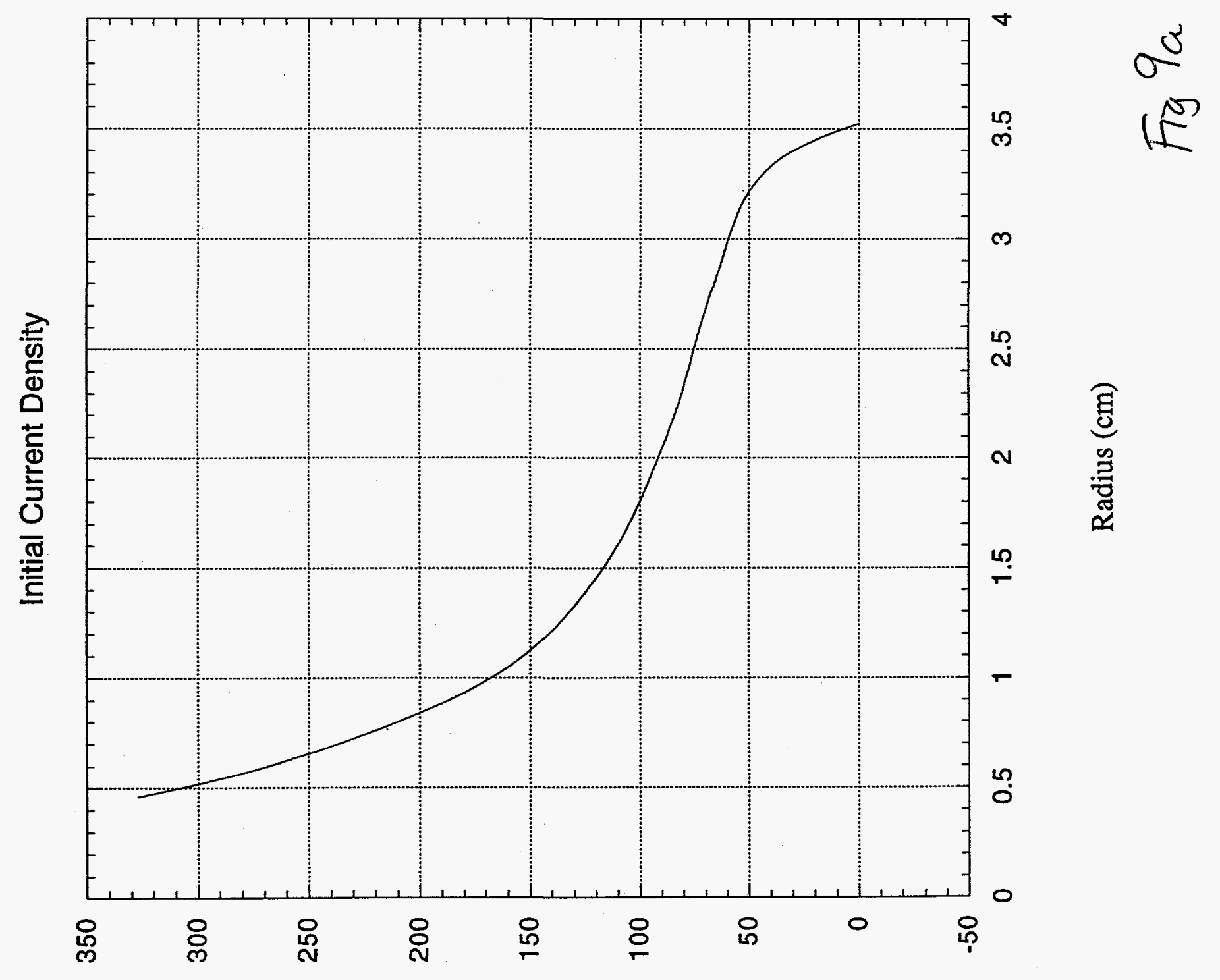

( 


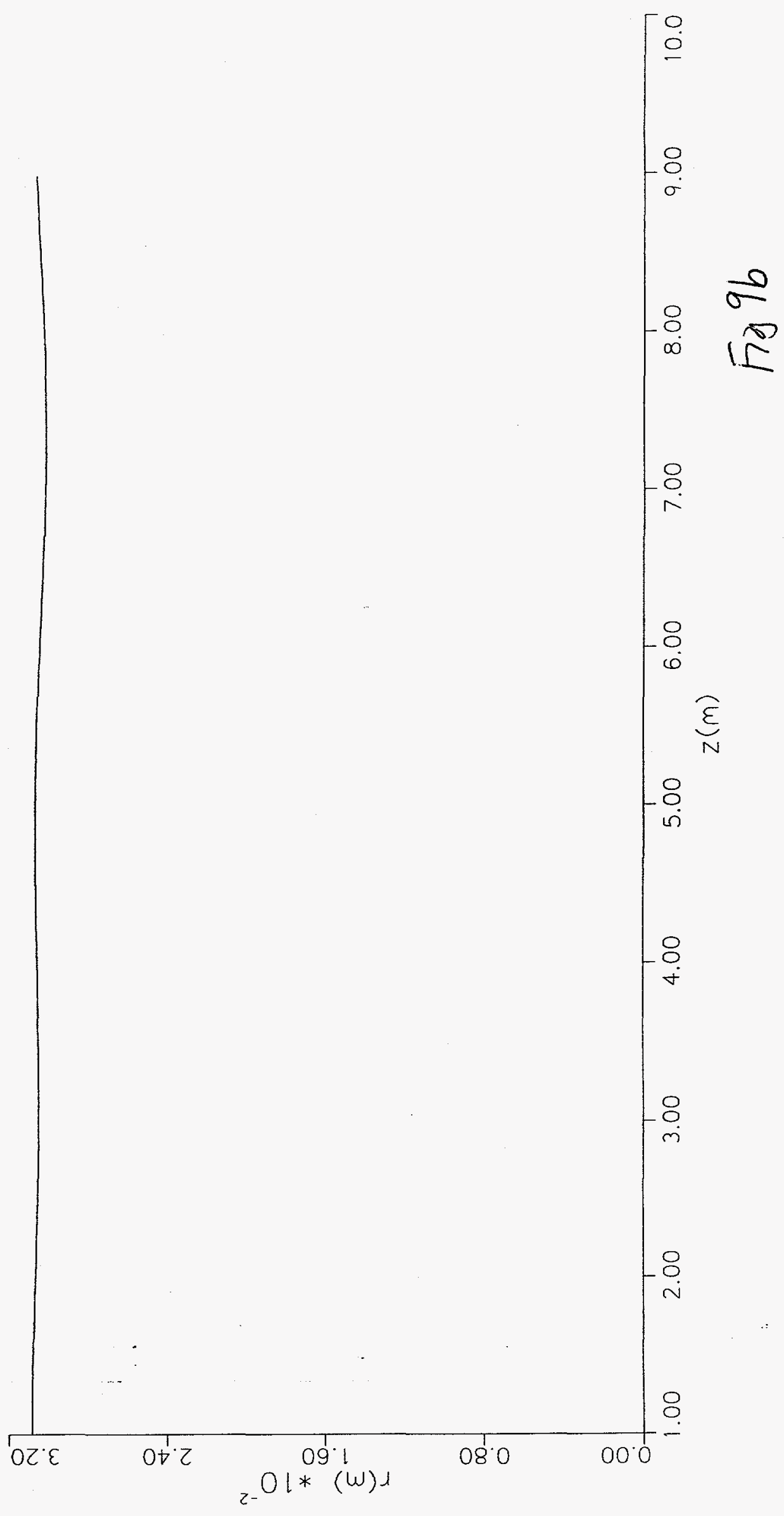




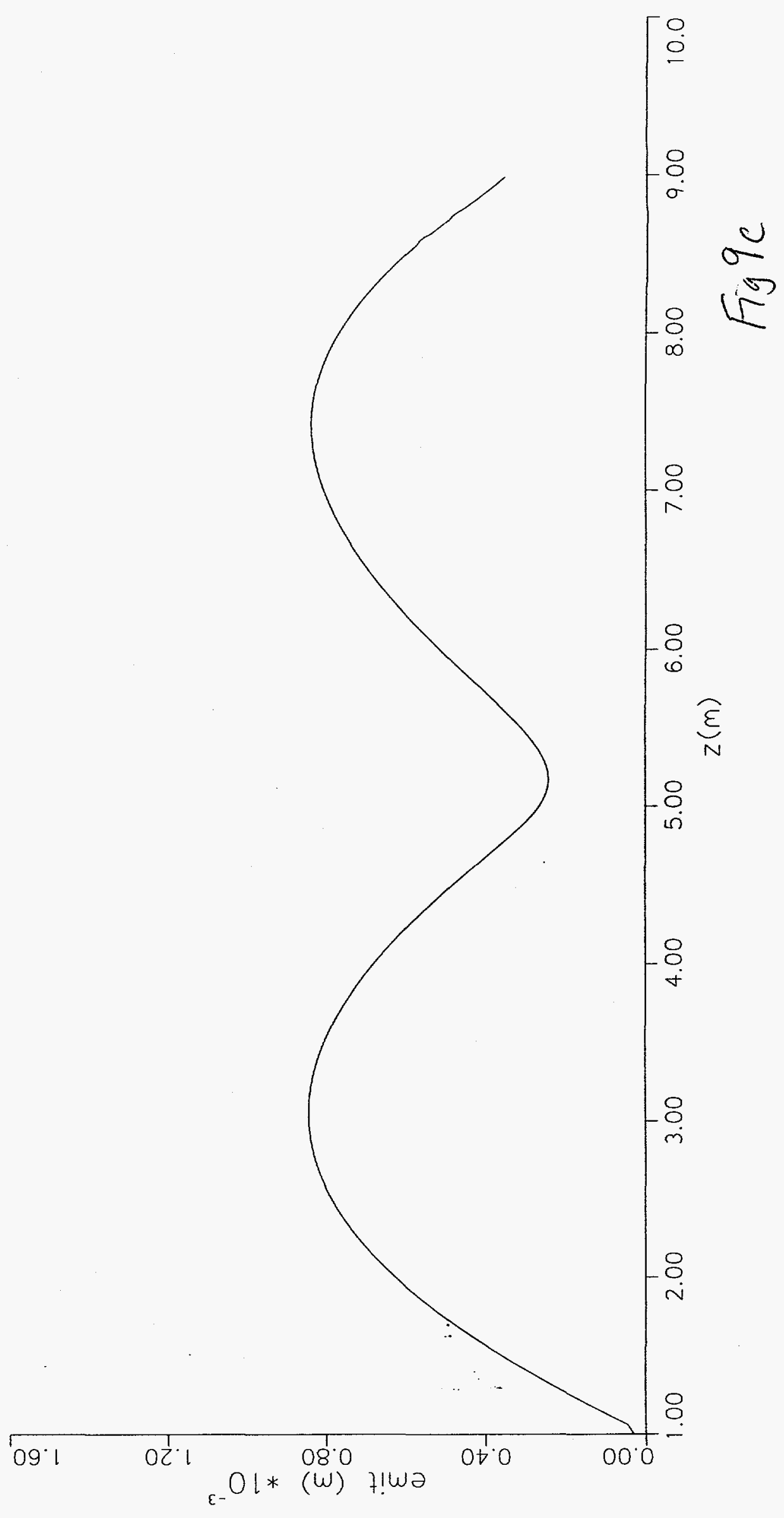




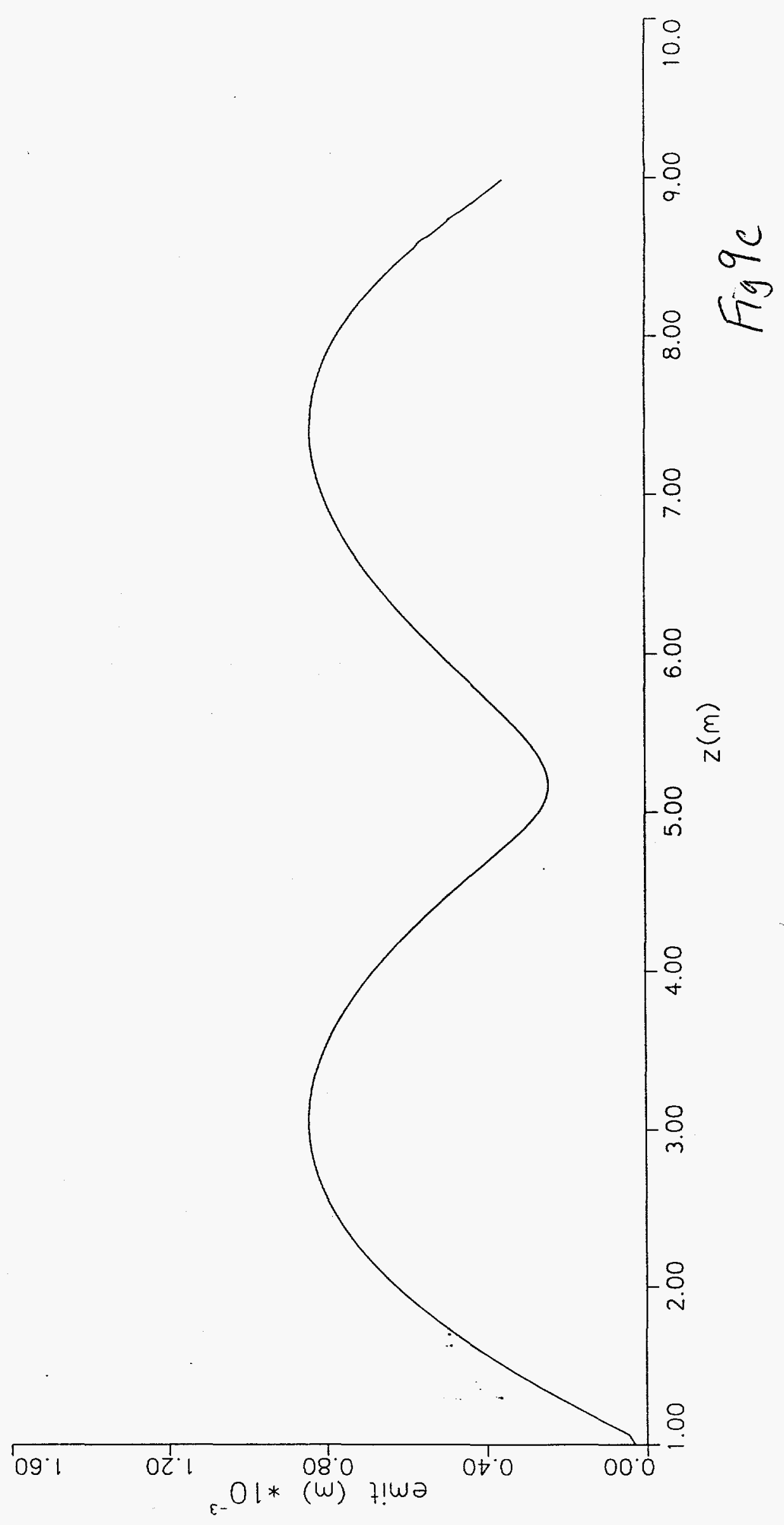

